\title{
Theoretical studies of photoexcitation and ionization in $\mathrm{H}_{2} \mathrm{O}$
}

\author{
G. H. F. Diercksen and W. P. Kraemer \\ Max Planck Institut für Astrophysik, Karl-Schwarzschild Strasse 1, 8046 Garching bei München, Federal \\ Republic of Germany \\ T. N. Rescigno and C. F. Bender \\ Theoretical Atomic and Molecular Physics Group, Lawrence Livermore Laboratory, University of California, \\ Livermore, California 94550 \\ B. V. McKoy \\ A. A. Noyes Laboratory of Chemical Physics, California Institute of Technology, Pasadena, California 91125
}

S. R. Langhoff

Computational Chemistry Group, NASA-Ames Research Center, Moffett Field, California 94035

\section{P. W. Langhoff}

Department of Chemistry, a) Indiana University, Bloomington, Indiana 47405 and Department of Theoretical Chemistry, University of Sydney, Sydney, N.S.W. 2006, Australia (Received 3 August 1981; accepted 28 August 1981)

\begin{abstract}
Theoretical studies are reported of the complete dipole excitation and ionization spectrum in $\mathrm{H}_{2} \mathrm{O}$ employing Franck-Condon and static-exchange approximations. Large Cartesian Gaussian basis sets are used to represent the required discrete and continuum electronic eigenfunctions at the ground-state equilibrium geometry, and previously devised moment-theory techniques are employed in constructing the continuum oscillator-strength densities from the calculated spectra. Detailed comparisons are made of the calculated excitation and ionization profiles with recent experimental photoabsorption studies and corresponding spectral assignments, electron impact-excitation cross sections, and dipole $(e, 2 e) /(e, e+i o n)$ and synchrotronradiation studies of partial-channel photoionization cross sections. The various calculated excitation series in the outer-valence $\left(1 b_{1}^{-1}, 3 a_{1}^{-1}, 1 b_{2}^{-1}\right)$ region are found to include contributions from valence-like $2 b_{2}\left(\sigma^{*}\right)$ and $4 a_{1}\left(\gamma^{*}\right)$ virtual orbitals, as well as appropriate $n s a_{1}, n p a_{1}, n d a_{1}, n p b_{1}, n p b_{2}, n d b_{1}, n d b_{2}$, and $n d a_{2}$ Rydberg states. Transition energies and intensities in the $\sim 7$ to $19 \mathrm{eV}$ interval obtained from the present studies are seen to be in excellent agreement with the measured photoabsorption cross section, and to provide a basis for detailed spectral assignments. The calculated $\left(1 b_{1}^{-1}\right) X^{2} B_{1},\left(3 a_{1}^{-1}\right)^{2} A_{1}$, and $\left(1 b_{2}^{-1}\right)^{2} B_{2}$ partial-channel cross sections are found to be largely atomic-like and dominated by $2 p \rightarrow k d$ components, although the $2 b_{2}\left(\sigma^{*}\right)$ orbital gives rise to resonance-like contributions just above threshold in the $3 a_{1} \rightarrow k b_{2}$ and $1 b_{2} \rightarrow k b_{2}$ channels. It is suggested that the latter transition couples with the underlying $1 b_{1} \rightarrow k b_{1}$ channel, accounting for a prominent feature in the recent high-resolution synchrotron-radiation measurements. When this feature is taken into account, the calculations of the three outer-valence channels are in excellent accord with recent synchrotron-radiation and dipole $(e, 2 e)$ photoionization cross-sectional measurements. The calculated innervalence $\left(2 a_{1}^{-1}\right)$ cross section is also in excellent agreement with corresponding measured values, although proper account must be taken of the appropriate final-state configuration-mixing effects that give rise to a modest failure of the Koopmans approximation, and to the observed broad PES band, in this case. Finally, the origins of the various spectral features present in the measured $1 a_{1}$ oxygen $K$-edge electron energy-loss profile in $\mathrm{H}_{2} \mathrm{O}$ are seen to be clarified fully by the present calculations.
\end{abstract}

\section{INTRODUCTION}

Photoprocesses in the water molecule are relevant to aspects of the aeronomical, astrophysical, and biological sciences. ${ }^{1}$ Recent refinements in VUV synchrotron-radiation, ${ }^{2,3}$ electron-impact excitation, ${ }^{4}$ and dipole $(e, 2 e) /(e, e+\text { ion })^{5}$ spectroscopy have provided highly reliable experimental determinations of cross sections for photoexcitation, ionization, and dissociation in this important compound. ${ }^{8 \rightarrow}$ In the present article, correspondingly reliable theoretical studies of the complete vertical-electronic dipole excitation and ionization spectrum in $\mathrm{H}_{2} \mathrm{O}$ are reported. The overall character of the observed spectrum and its prominent features are clarified on the basis of the present calcula-

\footnotetext{
2) Permanent address.
}

tions in terms of elementary molecular-orbital concepts. Particular attention is paid in this to contributions from $2 b_{2}$ and $4 a_{1}$ virtual valence orbitals to the spectrum, an aspect of photoprocesses in $\mathrm{H}_{2} \mathrm{O}$ that has apparently been the subject of some controversy. ${ }^{6-8}$

In order to discuss and clarify both the discrete and continuous spectra in $\mathrm{H}_{2} \mathrm{O}$ from the common perspective of molecular-orbital calculations, it is convenient to employ in the development Stieltjes-Tchebycheff techniques previously devised for this purpose. ${ }^{9110}$ A significant number of applications of this approach to molecular photoexcitation and ionization studies have been reported to date, largely in the one-electron verticalelectronic (Franck-Condon), separated-channel, staticexchange approximation. ${ }^{11,12}$ Methods have also been formulated for cases in which the effects of configura- 
tion interaction are of paramount importance in molecular photoionization, and detailed computational applications reported. ${ }^{13}$ In the present study of the electronic dipole spectrum in $\mathrm{H}_{2} \mathrm{O}$, separated-channel static-exchange calculations are found to provide generally satisfactory results, although there is also some evidence of channel coupling present in specific cases, as indicated below.

The calculated discrete excitation series converging on the $\left(1 b_{1}^{-1}\right)^{2} B_{1},\left(3 a_{1}^{-1}\right)^{2} A_{1}$, and $\left(1 b_{2}^{-1}\right)^{2} B_{2}$ ionic limits are seen to include contributions from Rydberg as well as $4 a_{1}$ and $2 a_{2}$ valence-like orbitals. Both the positions and intensities of the calculated series are in excellent accord with recent VUV synchrotron-radiation studies, and would seem to account satisfactorily for all of the features in the observed spectrum. ${ }^{2}$ Correspondingly, the calculated partial-channel photoionization cross sections for parent-ion production are in very good accord with the results of recent dipole $(e, 2 e)$ and synchrotronradiation studies. ${ }^{3,5}$ Resonance-like $2 b_{2}$ contributions are found in $k b_{2}$ final-state symmetry just above threshold in both $\left(3 a_{1}^{-1}\right)^{2} A_{1}$ and $\left(1 b_{2}^{-1}\right)^{2} B_{1}$ channels, the latter feature apparently autoionizing into the $\left(1 b_{1}^{-1}\right)^{2} B_{1}$ channel, and accounting for a prominent maximum appearing in recent synchrotron-radiation measurements of the corresponding partial cross section. ${ }^{3}$ When this channel coupling is taken into account, it is seen that the present calculations are in excellent accord with the measured outer-valence-shell partial-channel cross sections. ${ }^{3,5}$ Because the Koopmans approximation is generally valid in $\mathrm{H}_{2} \mathrm{O},{ }^{14-16}$ the separated-channel, static-exchange approximation gives cross sections in good accord with the measured values in the $2 a_{1}$ inner-valence region. ${ }^{5,17}$ However, the effects of final-ionic-state configuration mixing are required to account for the large width of the measured photoelectron band in this interval. ${ }^{14-17}$ Finally, the calculated $K$-edge excitation and ionization cross section accounts satisfactorily for all of the prominent features in the measured electron-impact spectrum. ${ }^{4}$ Comparisons are made throughout of the present comprehensive results with previously reported theoretical studies of the discrete and continuous spectra in $\mathrm{H}_{2} \mathrm{O} .{ }^{18-35}$

TABLE I. Dipole allowed excitation spectra in $\mathrm{H}_{2} \mathrm{O}{ }^{2}$

\begin{tabular}{lccc}
\hline Orbital (HFIP/EVIP) & ${ }^{1} A_{1} \rightarrow{ }^{1} B_{1}(x)$ & ${ }^{1} A_{1} \rightarrow{ }^{1} B_{2}(y)$ & ${ }^{1} A_{1} \rightarrow{ }^{1} A_{1}(z)$ \\
\hline $1 b_{1}(13.76 / 12.61) \rightarrow$ & $a_{1}$ & $a_{2}$ & $b_{1}$ \\
$3 a_{1}(15.41 / 14.73) \rightarrow$ & $b_{1}$ & $b_{2}$ & $a_{1}$ \\
$1 b_{2}(19.50 / 18.55) \rightarrow$ & $a_{2}$ & $a_{1}$ & $b_{2}$ \\
$2 a_{1}(37.02 / 32.2) \rightarrow$ & $b_{1}$ & $b_{2}$ & $a_{1}$ \\
$1 a_{1}(559.46 / 539.7) \rightarrow$ & $b_{1}$ & $b_{2}$ & $a_{1}$ \\
\hline
\end{tabular}

${ }^{a}$ Dipole allowed final-state symmetries in $\mathrm{H}_{2} \mathrm{O}$ for electricfield polarizations in the body frame in the $x$ (out of plane), $y$, and $z$ directions.

Values in parentheses refer to Hartree-Fock ionization potentials (HFIP) in $\mathrm{eV}$ calculated in a $(9 s, 5 p ; 4 s) /[4 s, 2 p ; 2 s]$ basis (Refs, 41 and 42 ) (total HF energy $=-76.0076$ a. u.), and the experimental vertical ionization potentials (EVIP) of Turner et al., (Ref. 15) and Siegbahn et al. (Ref. 14).
A brief discussion of the physical basis underlying the static-exchange approximation employed in the present development is presented in Sec. II. The discretebasis-set methodology used to solve the appropriate static-exchange equations is given in Sec. III, and the results of the calculations are reported and discussed in Sec. IV. Some concluding remarks are made in Sec. V.

\section{STATIC-EXCHANGE APPROXIMATION IN $\mathrm{H}_{2} \mathrm{O}$}

Photoelectron spectra in $\mathrm{H}_{2} \mathrm{O}$ exhibit five distinct bands that can be associated in the first (Koopmans) approximation with the removal of electrons from individual molecular orbitals having corresponding vertical ionization potentials $\left[1 b_{1}(12.61 \mathrm{eV}), 3 a_{1}(14.73 \mathrm{eV}), 1 b_{2}(18.55\right.$ eV) $2 a_{1}(32.2 \mathrm{eV})$, and $\left.1 a_{1}(539.7 \mathrm{eV})\right] .{ }^{14-17}$ Appropriate theoretical studies indicate the three outermost vertical ionic states are indeed Koopmans or single-hole like with two-hole one-particle configurations accounting for less than $\sim 5 \%$ of the total wave functions in each case. ${ }^{36}$ Similarly, the $K$-edge ionic state can be regarded as a strongly shifted Koopmans-like state, whereas there is some evidence that the inner-valence $2 a_{1}$ PES band at $32.2 \mathrm{eV}$ includes a number of satellite states in the 30 to $35 \mathrm{eV}$ interval. ${ }^{5,17,36}$ In all five cases, however, it is clear that single-hole or Koopmans states provide the appropriate potentials for calculations of vertical electronic excitation/ionization spectra, since the effects of relaxation and reorganization apparently play a small role in vertical ionization in $\mathrm{H}_{2} \mathrm{O}$.

Appropriate excitation and ionization spectra are obtained in the present development from conventional configuration-interaction or Ritz variational calculations employing singlet-coupled many-electron wave functions of proper symmetry. In the separated-channel approximation these are written in the forms $\mathrm{m}^{37-40}$

$$
k \Phi_{i}^{(\gamma)}=\left(\phi_{1} \phi_{2} \ldots k \phi_{i}^{(\gamma)} \cdots \phi_{N}\right),
$$

where the $\phi_{i}(i=1, N)$ are the occupied canonical groundstate Fock spin orbitals, the $i$ th orbital $\phi_{i}$ has been replaced $\left(\phi_{i}-k \phi_{i}^{(\gamma)}\right)$ by the excitation or continuum orbital $k \phi_{i}^{(\gamma)}$ to be determined, $\gamma$ is the channel label designating the final-state symmetry of excitation or ionization, and the parentheses indicate a singlet-coupled Slater determinant. The appropriate many-electron and orbital symmetries for dipole-allowed excitations in $\mathrm{H}_{2} \mathrm{O}$ corresponding to the three independent body-frame polarization directions are conveniently tabulated in Table I. The orbitals $k \phi_{i}^{(\gamma)}$ can be determined by expansion in the virtual Fock orbitals, $\phi_{\alpha}(\alpha>N)$, including the continuous portion of the spectrum, in conjunction with the Ritz principle

$$
\delta E\left[k \phi_{i}^{(\gamma)}\right]=\delta\left\langle k \Phi_{i}^{(\gamma)}|H| k \Phi_{i}^{(\gamma)}\right\rangle=0,
$$

where $H$ is the total many-electron Hamiltonian. Alternatively, carrying out the variation of Eq. (2) explicitly gives the familiar static-exchange equations ${ }^{39,40}$

$$
\left(h_{i}-\epsilon\right) k \phi_{i}^{(r)}=0
$$

for the excitation/ionization orbitals $k \phi_{l}^{\langle\gamma\rangle}$. Here $\epsilon$ refers to negative discrete or positive continum energies, and 


$$
h_{i}=T+V+V_{i}^{(N-1)}
$$

is the one-electron static-exchange Hamiltonian, with $T$ and $V$ kinetic and nuclear potential-energy operators, respectively, and $V_{i}^{(N-1)}$ the appropriate singlet-coupled static-exchange or hole potential obtained upon removal of an electron from the $i$ th occupied orbital. In the case of ground-state $\mathrm{H}_{2} \mathrm{O}$, the hole potentials take the form

$$
V_{i}^{(N-1)}=\hat{J}_{i}+\hat{K}_{i}+\sum_{j} 2 \hat{J}_{j}-\hat{K}_{j},
$$

where the sum in $j$ is over the Coulomb $\left(\hat{J}_{j}\right)$ and exchange $\left(\hat{K}_{j}\right)$ operators of the doubly occupied unexcited orbitals, and $\hat{J}_{i}$ and $\hat{K}_{i}$ refer to the orbital of the active or excited/ionized electron.

When solutions of Eqs. (1)-(5) are obtained, the vertical-electronic transition energies and oscillator strengths or densities are given by the familiar expressions

$$
\begin{aligned}
& \omega_{i}=\epsilon-\epsilon_{i}, \\
& d f_{i}^{(r)} / d \epsilon=(4 / 3) \omega_{i}\left|\left\langle k \phi_{i}^{(r)}|\mu| \phi_{i}\right\rangle\right|^{2},
\end{aligned}
$$

where $\phi_{i}$ and $\epsilon_{i}$ are the $i$ th canonical occupied orbital and energy, respectively, and the additional factor of 2 in Eq. (6b) arises from the use of singlet-coupled wave functions [Eq. (1)]. Since the vertical-electronic approximation is employed in Eqs. (1)-(6), the corresponding partial-channel cross sections

$$
\sigma_{y i}(\omega)(\mathrm{Mb})=109.75\left(d f_{i}^{(r)} / d \epsilon\right)\left(\mathrm{eV}^{-1}\right)
$$

implicitly invoke vibrational and rotational closure in the vertical-electronic Franck-Condon approximation. Consequently, comparisons with appropriate corresponding experimental measurements that implicitly sum over vibrational and rotational states are clearly in order.

When configuration mixing between channels is potentially important, Eqs. (6) and (7) are still appropriate, although the orbitals of Eqs. (1)-(5) are inadequate. In such cases it is necessary to include in the Ritz variational calculations [Eq. (2)] configurations in which two or more occupied orbitals are individually excited $\left(\phi_{i}\right.$ $\left.\rightarrow k \phi_{i}^{(r)}, \phi_{j} \rightarrow k \phi_{j}^{(0)}\right)$. In this way the excitation or ionization orbitals required in Eqs. (6) and (7) are determined in the presence of mixing with other singly excited configurations involving different occupied and virtual orbit als. This approach can be designated as the couple channel static-exchange or Tamm-Dancoff approximation.

\section{CALCULATIONS IN $\mathrm{H}_{2} \mathrm{O}$}

In order to obtain solutions of Eqs. (1)-(5) it is necessary to first construct a suitable Hartree-Fock groundstate wave function. This is accomplished in the present study at the experimental equilibrium geometry $\left[R(\mathrm{O}-\mathrm{H})=1.81 a_{0}, \theta(\mathrm{H}-\mathrm{O}-\mathrm{H})=104.5^{\circ}\right]^{41}$ employing a $(9 \mathrm{~s}$, $5 p ; 4 s) /[4 s, 2 p ; 2 s]$ Cartesian Gaussian basis set of standard type. ${ }^{41,42}$ The resulting total Hartree-Fock and canonical-orbital energies are shown in Table $I$, the latter in comparison with corresponding experimental values. ${ }^{14,15}$ It is seen that the HF or Koopmans values for the outer three valence orbitals are in general accord with measured values, whereas there are larger discrepancies in the two deeper-lying orbitals. The total Hartree-Fock energy is in accord with results obtained from comparable basis sets. ${ }^{41}$

Discrete-basis-set methodology is employed in the present development to obtain solutions of Eqs. (1)-(5) for both negative and positive energies. Large supplemental Gaussian basis sets are employed in conjunction with the valence basis used in the ground-state calcula tion in construction of virtual Fock states. These, in turn, are used to represent the static-exchange orbitals of Eqs. (1) and (5) employing conventional procedures. ${ }^{43}$

In Table II are shown supplemental basis sets used in conjunction with the valence basis in the present calculations in $\mathrm{H}_{2} \mathrm{O}$. The orbital exponents are chosen as extensions of those in the valence basis in order to form an even-tempered sequence having successive exponent ratios of $\sim 1,8$. It is seen that the basis sets, therefore, include very compact and very diffuse functions, approaching linear dependence in the interval. Such basis sets have been found previously to provide spectrally complete pseudostates in other molecules. ${ }^{11-13}$ Those static-exchange pseudostates lying below threshold provide approximations directly to the corresponding Rydberg or valence states, whereas those lying above threshold provide the information required in construction of the corresponding oscillator-strength density and

TABLE II. Supplemental Cartesian Gaussian basis sets employed in $\mathrm{H}_{2} \mathrm{O}$ pseudospectral calculations."

\begin{tabular}{lll}
\hline Type & Number & Exponent range \\
\hline & $a_{1}(46)$ symmetry & \\
$s$ & 8 & $0.158-0.001$ \\
$p_{z}$ & 8 & $0.119-0.001$ \\
$d_{x^{2}, d_{y}, d_{y^{2}}}$ & $12,12,12$ & $0.85-0.001$ \\
& $a_{2}(12)$ symmetry & \\
$d_{x y}$ & 12 & $0.85-0.001$ \\
& $b_{1}(21)$ symmetry & \\
$p_{x}$ & 8 & $0.119-0.001$ \\
$d_{x z}$ & 12 & $0.85-0.001$ \\
& $b_{2}(23)$ symmetry & \\
$p_{y}$ & 8 & $0.119-0.001$ \\
$d_{y z}$ & 12 & $0.85-0.001$ \\
\hline \hline
\end{tabular}

${ }^{2}$ Basis functions employed, in addition to the target $(9 s, 5 p ; 4 s) /$ $[4 s, 2 p ; 2 s]$ valence basis, in the solution of Eqs. (1)-(5). All functions are located on the oxygen nucleus.

Numbers in parentheses give the dimensionalities of resulting virtual Fock pseudospectra. In the case of $a_{1}$ symmetry the 46-term virtual spectrum obtained is arbitrarily truncated by deleting the six highest-lying virtual Fock orbitals obtained. Consequently, the separated-channel static-exchange pseudospectra obtained in this case are comprised of 40 terms each. cAn exponent ratio of 1.8 for successive values is employed in the indicated ranges. 
photo cross section [Eq. (7)] following the previously described moment methods. ${ }^{9,10}$

The computational methodology employed in the present development is the same for each particular excitation/ionization channel considered (Table I). A large one- and two-electron integral tape is first written over the entire list of valence and supplemental (Table II) basis orbitals. Smaller tapes are then constructed from this master upon which are written the integrals required in the construction of the pseudospectra for the individual channels of Table II. These smaller tapes are then transformed in the construction of virtual Fock symmetry orbitals appropriate for the final-state symmetry of interest. Single-excitation CI calculations are then performed and all roots and vectors are obtained, from which the transition frequencies and strengths of Eqs. (6) are constructed. Note that the development is the same in the cases of uncoupled and coupled-channel calculations, although the integral tapes and CI matrices are, of course, larger in the latter case.

\section{PHOTOEXCITATION AND IONIZATION IN $\mathrm{H}_{2} \mathrm{O}$}

In order to interpret the origins of the various features in the calculated and measured photo cross sections reported below, it is helpful to first recall the general natures of the occupied and virtual canonical orbitals in $\mathrm{H}_{2} \mathrm{O} .{ }^{44}$ The outer-valence region is comprised of $1 b_{1}(\pi), 3 a_{1}(\gamma)$, and $1 b_{2}(\sigma)$ orbitals which are largely of nonbonding $\pi_{x}$ type, angular or torsional $\gamma$ bonding type, and $\mathrm{O}-\mathrm{H} \sigma$-bonding type, respectively, whereas the inner valence $2 a_{1}(2 s)$ and $K$-edge $1 a_{1}(1 s)$ orbitals are largely $2 s$ and $1 s$ oxygen-like, respectively. There is also some evidence, however, of $\mathrm{O}-\mathrm{H}$ bonding character present in the $2 a_{1}$ orbital. The virtual spectrum will be comprised of $4 a_{1}\left(\gamma^{*}\right)$ and $2 b_{2}\left(\sigma^{*}\right)$ antibonding valence-like orbitals, as well as more diffuse molecular orbitals of good symmetry type made up of $s, p, d$, and higher angular momentum atomic Rydberg orbitals. The continuous region of the orbital spectrum will include Coulombic type functions that are natural continuations of the Rydberg series, as well as possible contributions from the valence $4 a_{1}\left(\gamma^{*}\right)$ and $2 b_{2}\left(\sigma^{*}\right)$ orbitals indicated above.

In the following subsections the calculated photoexcitation and ionization spectra in $\mathrm{H}_{2} \mathrm{O}$ in the outer-valence $\left(1 b_{1}, 3 a_{1}, 1 b_{2}\right)$, inner-valence $\left(2 a_{1}\right)$, and $K$-edge $\left(1 a_{1}\right)$ regions are described and discussed, and comparisons are made with corresponding experimental and previously reported theoretical studies.

\section{A. Valence region in $\mathrm{H}_{2} \mathrm{O}$}

In Fig. 1 is given a semiquantitative overview of the calculated separated-channel series associated with excitation of electrons from the three outermost orbitals in $\mathrm{H}_{2} \mathrm{O}$. Also shown in the figure is the total photo cross section obtained from synchrotron-radiation measurements that achieve $\sim 0.03 \AA$ spectral resolution. ${ }^{2}$ More detailed quantitative descriptions of the calculated excitation series and photoionization cross sections in the separated-channel approximation are given in Tables III

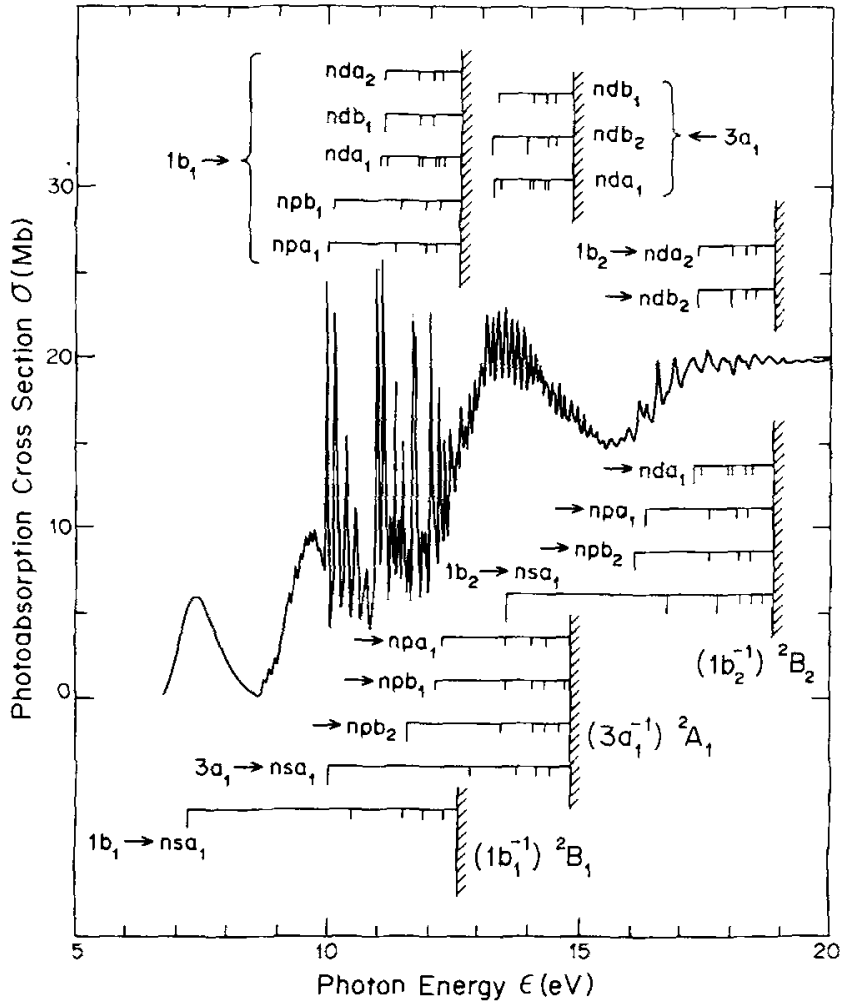

FIG. 1. Outer-valence-shell photoabsorption cross section in $\mathrm{H}_{2} \mathrm{O}$; experimental values from the synchrotron radiation measurements of P. Gürtler, V. Saile, and E. E. Koch (Ref. 2); theoretical values and assignments from separated-channel static-exchange (IVO) calculations employing the basis sets of Table II as described in the text; intensity scale: strong, $f$ $>0.1$; medium, $0.1>f>0.01$; weak, $f<0.01$.

to VI and Figs. 2 to 4 , respectively. As in previous studies, ${ }^{11,12}$ the calculated discrete excitation series are made to converge on the experimentally observed, ${ }^{14,15}$ rather than Hartree-Fock, vertical-ionization potentials (Table I).

\section{1. $1 b_{1}\left(I . P_{.}=12.61 \mathrm{eV}\right)$ spectrum}

It is seen from Table III that well-defined $1 b_{1} \rightarrow n s a_{1}$, $n p a_{1}, n d a_{1} ; n p b_{1}, n d b_{1} ;$ and $n d a_{2}$ series are obtained from the calculations, in very good accord with the assignments based on measured values also shown in the table. Note that the calculations include mixing among the various Rydberg states of given molecular orbital symmetry, but that the series nevertheless separate into $s, p$, and $d$ components having approximate quantum defects of 1.4,0.7, and 0, respectively. Quantum-defect and Coulomb estimates of transition energies and $f$ numbers, respectively, obtained using these values are also shown in Table III to aid in the series identification. ${ }^{8,45}$ The various calculated $1 b_{1}$ excitations are in accord with some of the previously reported theoretical studies, ${ }^{18-30}$ although in many cases these do not employ sufficient numbers of the compact and diffuse basis functions (Table II) required to satisfactorily describe the relevant states (see Table VI). 
TABLE III. $1 b_{1}$ (I. P. $=12.61 \mathrm{eV}$ ) excitation spectra in $\mathrm{H}_{2} \mathrm{O}$.

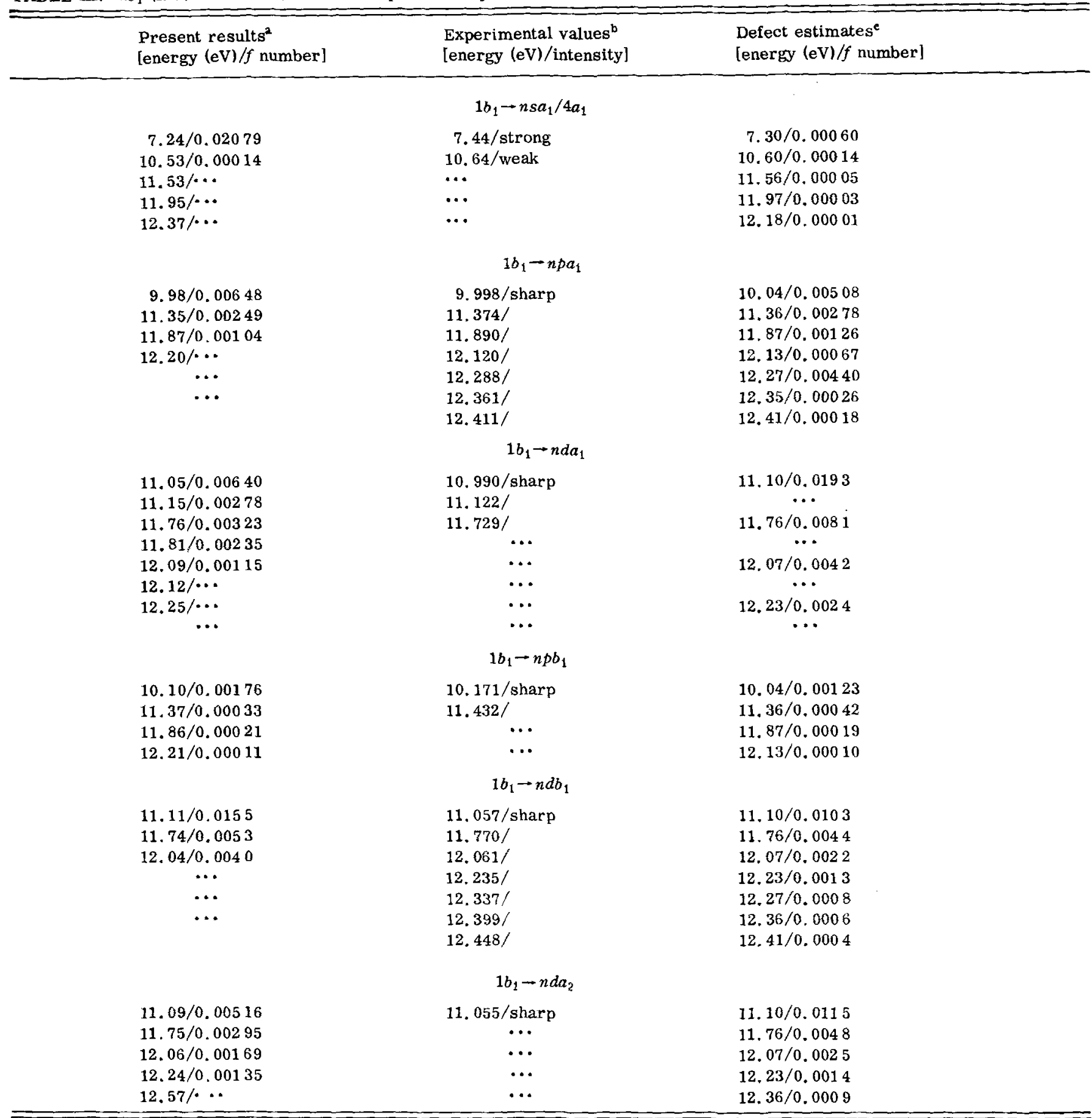

${ }^{2}$ Vertical excitation energies obtained from the separated-channel static-exchange development of Eqs. (1)-(7) empioying the basis sets of Table II. The values shown are made to converge on the experimentally observed vertical ionization potential of $12.61 \mathrm{eV}$ (Ref. 15). In the case of $1 b_{1}-n d a_{1}$ excitations, two separate $n d\left(z^{2}\right)$ and $n d\left(x^{2}-y^{2}\right)$ series are obtained. Previously reported calculations of the lowest few excitations in various approximations [Refs. 18-30] employing smaller basis sets than those of Table II are in general accord with the present results (see Table VD.

${ }^{b}$ Experimental positions taken from the measurements of P. Gürtler, V. Saile, and E. E. Koch (Ref. 2). See also H. -t. Wang, W. S. Felps, and S. P. McGlynn (Ref. 6). Approximate intensity designations are made here on the basis of the measured cross section. The higher members of the measured $n p$ and $n d$ series are here assigned as $1 b_{1} \rightarrow n p a_{1}$ and $n d b_{1}$ excitations, respectively, on the basis of calculated intensities. Although the latter assignment is in accord with Wang et al. (Ref. 6), the former is not.

${ }^{\circ}$ Quantum-defect estimates obtained for $n=3,4, \ldots$ from the Rydberg formula $\left[\epsilon_{n}=\epsilon_{t}-13.6 \mathrm{eV} /(n-\delta)^{2}\right]$ and Coulomb approximation $\left[f_{n}=g\left(\epsilon_{t}\right) /(n-\delta)^{3}\right]$, where $\delta$ is the appropriate defect and $g\left(\epsilon_{t}\right)$ is the photoionization oscillator-strength density at threshold in Hartree a, u. [Fig. 2(a)]. Procedures employed in separating the total $g\left(\epsilon_{t}\right)$ values into the contributions from the various Rydberg series discussed in the text give $1 b_{1} \rightarrow k a_{1} ; k s-0.0, k p-0.1, k d-0.52 ; 1 b_{1} \rightarrow k b_{1}, k p-0.015, k d-0.28 ; 1 b_{1} \rightarrow k a_{2}$, $k d-0.31$, all in a.u. The defects employed are $\delta=1.40,0.7$, and 0 for $n s, n p$, and $n d$ series, respectively, in general accord with the corresponding oxygen-atom values of $\delta=1.20,0.76$, and 0.03 (Ref. 8 ). In the case of the $n d a_{1}$ excitations, the indicated quantum-defect values are appropriate for both $n d\left(z^{2}\right)$ and $n d\left(x^{2}-y^{2}\right)$ series. 
The (1.40) defect of the $1 b_{1}-n s a_{1}$ series is somewhat larger than the corresponding free oxygen-atom value of $1.2,{ }^{8}$ and the $f$ number of the resonance $(n=3)$ transition is significantly larger than that obtained from the Coulomb approximation, suggesting that the $4 a_{1}\left(\gamma^{*}\right)$ valence orbital perturbs the entire series. This observation is verified by examination of the calculated static-exchange orbitals, which are seen to include admixtures of the $4 a_{1}\left(\gamma^{*}\right)$ orbital. Note, however, that only the lowest $(n=3)$ member of the $1 b_{1} \rightarrow n s a_{1} / 4 a_{1}\left(\gamma^{*}\right)$ series has a substantial $f$ number, a situation clarified by the significantly larger $4 a_{1}\left(\gamma^{*}\right)$ contribution to the former than to the higher-lying members of the series. The resonance transition is known to photodissociate into $\mathrm{OH}\left(X^{2} \Pi\right)+\mathrm{H}\left({ }^{2} S\right)$ radical $f$ ragments, ${ }^{8}$ accounting for the broad, diffuse nature of the band (Fig. 1). Presumably, a diabatic dissociative surface associated with the $1 b_{1}^{-1} 4 a_{1}\left(\gamma^{*}\right)$ configuration crosses the $1 b_{1}-n s a_{1}$ Rydberg series in the immediate vicinity of the ground-state vertical Franck-Condon region. It is perhaps remarkable that there has been little discussion of the intravalence nature of this strong dissociative transition in $\mathrm{H}_{2} \mathrm{O}^{6-8}$

The $1 b_{1} \rightarrow n p a_{1}$ and $n p b_{1}$ series of Fig. 1 and Table III are seen to be largely Rydberg in character, with defects of $\sim 0.7$, in good accord with the corresponding free oxygen-atom value of $0.76 .^{8}$ Moreover, the calculations are in good agreement with the measured positions and intensities, confirming the spectral assignments in these cases. The higher members of the measured $n p$ series are given the assignment $1 b_{1} \rightarrow n p a_{1}$ on the basis of the larger calculated $f$ numbers relative to the $1 b_{1}-n p b_{1}$ series.

Evidently, four distinct $1 b_{1}-n d$ series, i. e., $1 b_{1}$ $-n d\left(z^{2}\right) a_{1}$, $n d\left(x^{2}-y^{2}\right) a_{1}, n d b_{1}$, and $n d a_{2}$, all having quantum defects of $\sim 0$, are obtained from the calculations. These are seen to be largely Rydberg in character, and are in generally good agreement with the positions and intensities of the four assigned nd series. ${ }^{2,48}$ Note that, as might be expected, the $f$ numbers of the nd series are uniformly larger than those of the corresponding $n p a_{1}$ and $n p b_{1}$ series.

With the exception of strong broad structures at $\sim 9.7$ and $11.5 \mathrm{eV}$, which are discussed separately further below, the calculated discrete series of Table III are seen to account for all of the spectral features below the $\left(1 b_{1}^{-1}\right)^{2} B_{1}$ threshold $(12.61 \mathrm{eV})$ in the measured photoabsorption cross section (Fig. 1).

In Fig. 2(a) are shown the $1 b_{1} \rightarrow k a_{1}, k a_{2}$, and $k b_{1}$ contributions to the $\left(1 b_{1}^{-1}\right)^{2} B_{1}$ partial-channel photoionization cross section in $\mathrm{H}_{2} \mathrm{O}$, as well as the sum of these in comparison with measured values. ${ }^{3,5}$ It is seen that the $1 b_{1}-k b_{1}$ and $k a_{2}$ contributions are indistinguishable from one another, and that the $1 b_{1}-k a_{1}$ component is approximately twice these values. The former observation is understood by noting that the $1 b_{1}(\pi)$ orbital is purely atomic oxygen $2 p_{x}$ in character, and that the $k p_{x} b_{1}$ orbital contributions (Table II) to $1 b_{1}-k b_{1}$ ionization are expected to be negligible, with the threshold cross section for $1 b_{1}-k p_{x} b_{1}$ ionization estimated (Table III) to be an order of magnitude smaller than $1 b_{1}-k d b_{1}$.

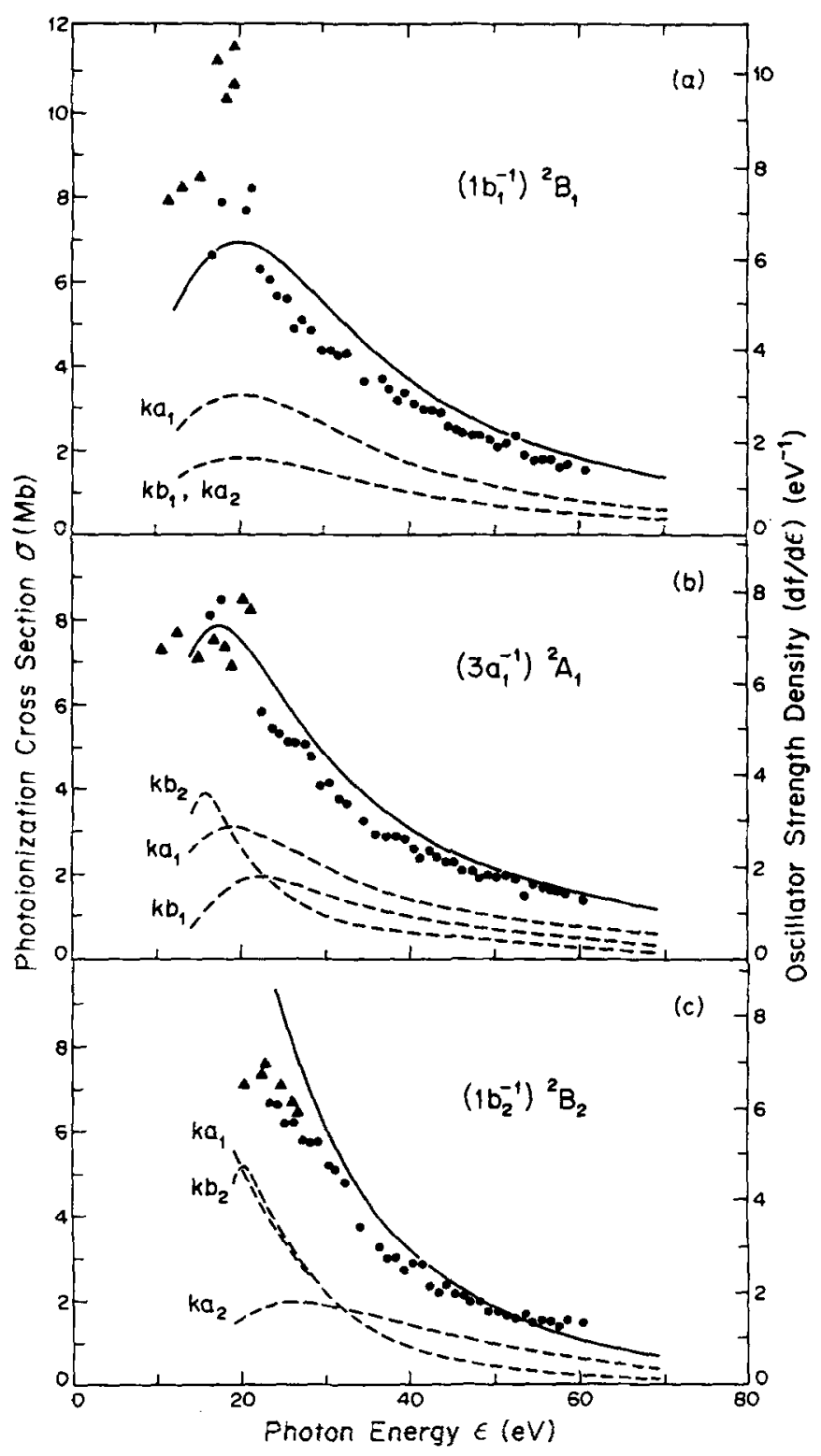

FIG. 2. Outer-valence-shell partial-channel photoionization cross sections in $\mathrm{H}_{2} \mathrm{O}$. (a) $\left(1 b_{1}^{-1}\right)^{2} B_{1}$ cross section; (-) vertical-electronic static-exchange calculations; (- - - ) static-exchange polarization components as indicated; $(\bullet)$ dipole $(e, 2 e)$ measurements of K. H. Tan, C. E. Brion, Ph. E. Van der Leeuw, and M. J. Van der Wiel (Ref. 5); ( 4 synchrotronradiation measurements of $O$. Dutuit, $K$. Ito, A. TabcheFouhaile, P. Morin, T. Baer, P. M. Guyon, and I. Nenner (Ref. 3); (b) As in (a) for $\left(3 a_{1}^{-1}\right)^{2} A_{1}$ ionization, (c) as in (a) for $\left(1 b_{2}^{-1}\right)^{2} B_{2}$ ionization.

Consequently, both $1 b_{1}-k b_{1}$ and $k a_{2}$ cross sections are purely $2 p_{x} \rightarrow k d_{x k}$ and $k d_{x y}$ atomic-like, respectively, in nature, and therefore can be expected to be identical. Similarly, the $1 b_{1} \rightarrow k d a_{1}$ component is seen from Table III to dominate the $k a_{1}$ contribution. In this case, however, both $k d z^{2} a_{1}$ and $k d\left(x^{2}-y^{2}\right) a_{1}$ contributions are present, giving rise to a cross section approximately twice those for the two other ( $x$ and $y$ ) polarization components.

The calculated $\left(1 b_{1}^{-1}\right)^{2} B_{1}$ separated-channel static-ex- 
change cross section is evidently in good agreement with the dipole $(e, 2 e)$ measurements, whereas there is a prominent feature at $\sim 18$ to $20 \mathrm{eV}$ in the synchrotronradiation measurements not present in the calculated profile. It is unlikely that a feature of this nature can arise as a consequence of static-potential shape-resonance effects. Rather, as is discussed further below, autoionization of states converging upon higher limits are more likely candidates, with $1 b_{2} \rightarrow 3 s a_{1} / 4 a_{1}$ and $2 b_{2}$ excitations favored, in particular. When this feature is accounted for in such a manner, as is discussed further below, it is seen that the measured values for the $\left(1 b_{1}^{-1}\right)^{2} B_{1}$ partial cross section are in good agreement with the present calculations. The corresponding $(e, e$ + ion) measurements, ${ }^{5}$ as well as PES band shapes, ${ }^{15,16}$ suggest the $\left(1 b_{1}^{-1}\right)^{2} B_{1}$ cross section results in the production of $\mathrm{H}_{2} \mathrm{O}^{+}$parent ions only, ${ }^{5}$ with no evidence of radical fragments, in general accord with the largely nonbonding character of the $1 b_{1}(\pi)$ orbital.

\section{2. $3 a_{1}(I . P .=14.73 \mathrm{eV})$ spectrum}

In Table IV are shown the calculated $3 a_{1} \rightarrow n a_{1}, n b_{2}$, and $n b_{1}$ excitations, which are seen to separate into welldefined $n s a_{1}, n p a_{1}$, nda $a_{1} ; n p b_{2}, n d b_{2} ;$ and $n p b_{1}, n d b_{1}$ series, respectively. The $3 a_{1}-n s a_{1}$ series contains a $3 s a_{1} / 4 a_{1}$ partially intravalence resonance member at $\sim 9.9 \mathrm{eV}$ that is seen to be stronger than the defect estimate, whereas the higher members of the series are weak with defect-like $f$ numbers $(\delta=1.4)$, a behavior in accord with the $1 b_{1} \rightarrow n s a_{1}$ series discussed above. The position and intensity of the resonance in this case, which is known to dissociate into $\mathrm{OH}\left(A^{2} \Sigma^{+}\right)$ $+\mathrm{H}\left({ }^{2} S\right)$ radical fragments, ${ }^{8}$ is in excellent agreement with measured values, ${ }^{2,7}$ whereas the higher members of the series are apparently too weak to be observed.

Of the three calculated $n p$ series $\left(3 a_{1}-n p a_{1}, n p b_{1}\right.$, and $\left.n p b_{2}\right)$, the first two are seen to be purely Rydberg in character $(\delta=0.7)$, whereas the resonance $f$ number in the $3 a_{1}-n p b_{2}$ series is significantly larger than the corresponding defect estimate. Examination of the appropriate orbitals reveals the presence of contributions from the $2 b_{2}\left(\sigma^{*}\right)$ valence orbital, accounting for the large $f$ number and the significantly lower position in the spectrum of this transition. Careful examination of the measured photo-cross section of Fig. 1 suggests the presence of a broad feature at $\sim 11.5 \mathrm{eV}$ underlying the Rydberg series converging on the $\left(1 b_{1}^{-1}\right)^{2} B_{1}$ limit which can be given the designation $3 a_{1} \rightarrow 3 p b_{2} / 2 b_{2}\left(a^{*}\right)$ on the basis of the present calculations. This feature has apparently not been previously assigned in the discrete spectrum of $\mathrm{H}_{2} \mathrm{O} .^{6-\beta}$ Because the weaker higher members of the $3 a_{1}$ $-n p b_{2}$ series, and of the $n p a_{1}$ and $n p b_{1}$ series, overlap with other strong transitions (Fig. 1), detailed spectral assignments are not possible in these cases. However, the positions of the experimentally assigned $3 a_{1} \rightarrow 4 p(13.5$ $e V), 5 p(14.1 \mathrm{eV})$ excitations are in general accord with the calculated values. The presence of vibrational structure in the strong feature in the $\sim 13$ to $14 \mathrm{eV}$ interval of the photo cross section similar to that observed in the $3 a_{1}^{-1}$ PES band ${ }^{25}$ can be attributed to intensity borrowing of the weak $3 a_{1} \rightarrow n p$ series from the strong $1 b_{2} \rightarrow 3 s a_{1} / 4 a_{1}$ intravalence transition (see below).
Of the four calculated $3 a_{1}-n d$ series $\left[3 a_{1}-n d\left(z^{2}\right) a_{1}\right.$, $n d\left(x^{2}-y^{2}\right) a_{1}$, nd $b_{2}$, and $\left.n d b_{1}\right]$, the $n d\left(z^{2}\right) a_{1}$ and $n d b_{2}$ transitions are seen to be relatively strong and to overlap to a considerable degree. The two resonance transitions in these series account for a portion of the strong feature centered at $\sim 13.5 \mathrm{eV}$ in the photo cross section (Fig. 1), which is seen to exhibit a vibrational structure similar to that of the measured $\left(3 a_{1}^{-1}\right)^{2} A_{1}$ PES band. ${ }^{15,16}$ The higher members of these two series, as well as the members of the weaker $3 a_{1}-n d\left(x^{2}-y^{2}\right) a_{1}$ and $n d b_{1}$ Rydberg series, are presently unassigned.

In Fig. 2(b) are shown the calculated $3 a_{1} \rightarrow k a_{1}, k b_{1}$, and $k b_{2}$ photoionization components and the total $\left(3 a_{1}^{-1}\right)^{2} A_{1}$ partial cross section in comparison with measured values. $^{3.5}$ It is seen that the $3 a_{1}-k a_{1}$ and $k b_{1}$ components are somewhat similar to the $1 b_{1} \rightarrow k a_{1}$ and $k b_{1}$ profiles of Fig. 2(a). This is a consequence of the similarity of $2 p$ orbital natures of the $3 a_{1}$ and $1 b_{1}$ molecular orbitals. However, the presence of $2 s$ hybridization and $1 s \mathrm{H}$ atom contributions in the $3 a_{1}$ orbital expand it somewhat spatially, resulting in ionization profiles that are peaked at lower energies than in the corresponding $1 b_{1}\left(-k a_{1}\right.$ and $\left.k b_{1}\right)$ cases. In contrast to these, the $3 a_{1} \rightarrow k b_{2}$ profile of Fig. 2(b) is seen to contain a prominent feature just above threshold that can be attributed to contributions from the $2 b_{2}\left(0^{*}\right)$ valence orbital. This orbital also contributes to the resonance transition in the $3 a_{1}$ $\rightarrow n p b_{2}$ series (Table IV), suggesting the presence of a diabatic $\left(3 a_{1}^{-1} 2 b_{2}\right)$ surface that cuts rather sharply through the Franck-Condon region in this case, resulting in a splitting of intensity between the discrete and continuous regions of the spectrum. A similar situation is observed in the $1 \pi_{g} \rightarrow n \sigma_{u} / k \sigma_{u}$ cross section in $\mathrm{O}_{2} \cdot{ }^{47}$ Evidently, the calculated $\left(3 a_{1}^{-1}\right)^{2} A_{1}$ cross section is in very good agreement with the measured $(e, 2 e)$ and synchrotronradiation values. ${ }^{3,5}$ Corresponding $(e, e+$ ion) measurements, as well as the PES band shapes, ${ }^{15,16}$ suggest that the $\left(3 a_{1}^{-1}\right)^{2} A_{1}$ channel results in production of $\mathrm{H}_{2} \mathrm{O}^{+}$parent ions only, in accordance with the largely angular bonding nature of the $3 a_{1}(\gamma)$ orbital.

\section{3. $1 b_{2}(1 . P .=18.55 \mathrm{eV})$ spectrum}

The calculated $1 b_{2}-n a_{1}, n b_{2}$, and $n a_{2}$ excitations of Table IV are seen to separate into well-defined $n s a_{1}$, $n p a_{1}, n d a_{1} ; n p b_{2}, n d b_{2} ;$ and $n d a_{2}$ series. The resonance in the $n s a_{1}$ series is the strongest feature in the entire spectrum, and apparently accounts for much of the broad strong band centered at $\sim 13.5 \mathrm{eV}$ in the measured cross section. ${ }^{2}$ This assignment is in general accord with Gürtler et al., ${ }^{2}$ who place the resonance at $\sim 13.8$ $\mathrm{eV}$, apparently on basis of quantum-defect estimates. As indicated above, the near degeneracy of the strong $1 b_{2}-3 s a_{1} / 4 a_{1}$ resonance with weaker $3 a_{1} \rightarrow n p$ and $n d$ excitations suggests configuration mixing in the $\sim 13$ to $14 \mathrm{eV}$ interval, presumably accounting for the $3 a_{1}^{-1} \mathrm{PES}$ band vibrational structure in the measured photoabsorption cross section. The measured ionization curve suggests formation of neutral fragments in this interval, ${ }^{7}$ in accord with the dissociative character of the diabatic $1 b_{2}^{-1} 4 a_{1}$ configuration. The calculated positions of the higher members of the series are also in general agreement with the experimental assignments. The second 
TABLE IV. $3 a_{1}$ (I. P. $=14.73 \mathrm{eV}$ ) and $1 b_{2}$ (I. P. $=18.55 \mathrm{eV}$ ' excitation spectra in $\mathrm{H}_{2} \mathrm{O}$.

\begin{tabular}{llll}
\hline $\begin{array}{l}\text { Present results } \mathrm{s}^{\mathrm{a}, \mathrm{b}} \\
\text { [energy }(\mathrm{eV}) / f \text { number] }\end{array}$ & $\begin{array}{l}\text { Defect estimates } \\
\text { [energy }(\mathrm{eV}) / f \text { number] }\end{array}$ & $\begin{array}{l}\text { Present results } \\
\text { [energy }(\mathrm{eV}) / f \text { number] }\end{array}$ & $\begin{array}{l}\text { Defect estimates } \\
\text { [energy }(\mathrm{eV}) / f \text { number] }\end{array}$ \\
\hline
\end{tabular}

$\begin{array}{rr} & 3 a_{1} \rightarrow n s a_{1} / 4 a_{1} \\ 9.88 / 0.0670 & 9.42 / 0.0381 \\ 12.77 / 0.0069 & 12.72 / 0.0089 \\ 13.67 / 0.0032 & 13.68 / 0.0033 \\ 14.07 / 0.0014 & 14.09 / 0.0016 \\ 14.33 / \cdots & 14.30 / 0.0009\end{array}$

$$
3 a_{1} \rightarrow n p a_{1}
$$

$12.24 / 0.000185$

$13.51 / 0.000091$

13. $99 \%$...

$14.30 / .$.
$12.16 / 0.000369$

13. $48 / 0.000091$

13. $99 / 0.000041$

14. $25 / 0.000022$

$$
3 a_{1} \rightarrow n d a_{1}
$$

$13.14 / 0.0133$

$13.23 / 0.0094$

$13.85 / 0.0045$

$13.90 / 0.0030$

$14.19 / 0.0035$

$14.24 / 0.0001$

$11.53 / 0.03763$

$13.35 / 0.00266$

$13.97 / 0.00090$

$14.25 / 0.00039$

$14.54 \%$..

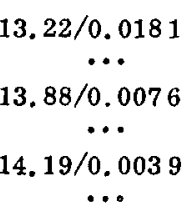

$3 a_{1} \rightarrow n p b_{2} / 2 b_{2}$

$12.16 / 0.00518$

$13.48 / 0.00175$

$13.99 / 0.00079$

$14.25 / 0.00042$

$14.39 / 0.00025$

$$
3 a_{1} \rightarrow n d b_{2}
$$

$12.16 / 0.00214$
$13.13 / 0.0315$

$13.86 / 0.0157$

$14.19 / 0.0080$

14. $39 / 0.0043$

$14.76 / \ldots$

$$
3 a_{1} \rightarrow n p b_{1}
$$

12. 08/0.00194

$13.45 / 0.00067$

$13.97 / 0.00031$

$14.23 / 0.00018$

$14.67 / \cdots$

13. $22 / 0.0266$

13. 88/0.0112

$14.19 / 0.0057$

14. $35 / 0.0033$

$14.45 / 0.0021$

$13.48 / 0.00072$

13. $99 / 0.00033$

14. $25 / 0.00018$

$14.39 / 0.00010$

$3 a_{1} \rightarrow n d b_{1}$

$13.25 / 0.00400$

$13.88 / 0.00235$

$14.18 / 0.00137$

14. $35 / 0.00107$

14. $75 / \cdots$

$1 b_{2} \rightarrow n s a_{1} / 4 a_{1}$

$\begin{array}{ll}13.23 / 0.1755 & 13.24 / 0.1956 \\ 16.47 / 0.0436 & 16.54 / 0.0579 \\ 17.44 / 0.0165 & 17.50 / 0.0172 \\ 17.86 / 0.0083 & 17.91 / 0.0082 \\ 18.10 \% \cdots & 18.12 / 0.0046 \\ 18.28 / \cdots & 18.24 / 0.0028\end{array}$

$1 b_{2} \rightarrow n p a_{1}$

$15.97 / 0.0142 \quad 15.98 / 0.0127$

$17.28 / 0.0044 \quad 17.30 / 0.0043$

$17.79 / 0.0014 \quad 17.81 / 0.0020$

$18.05 / \cdots 118.07 / 0.0010$

$1 b_{2} \rightarrow n d a_{1}$

$16.95 / 0.01200$

$17.10 / 0.00019$

$17.67 / 0.00870$

$17.73 / 0.00013$

$18.00 / \cdots$

$18.19 / \cdots$

$17.04 / 0.0147$

...

17. 70/0.0062

...

$18,01 / 0.0032$

...
15. $77 / 0.0187$

17. $27 / 0.0046$

17. $81 / 0.0023$

$18.07 / 0.0011$

$1 b_{2} \rightarrow n p b_{2} / 2 b_{2}$

$15.98 / 0.0143$

17. $30 / 0.0048$

$17.81 / 0.0022$

$18.07 / 0.0012$

$1 b_{2} \rightarrow n d b_{2}$

$17.04 / 0.0271$

17. $70 / 0.0132$

$18.01 / 0.0080$

$18.19 / 0.0043$

17. 04/0. 0344

17. $70 / 0.0145$

$18.01 / 0.0074$

$18.17 / 0.0043$

$1 b_{2} \rightarrow n d a_{2}$

$17.03 / 0.0066$

$17.70 / 0.0037$

18.01/0.0021

$18.18 / 0.0017$
$17.04 / 0.0139$

$17.70 / 0.0059$

$18.01 / 0.0030$

$18.17 / 0.0017$

As in Table III, footnote a, employing the indicated experimentally observed vertical ionization potentials (Ref. 15).

Experimental values for $3 a_{1} \rightarrow 3 s(9.85 \mathrm{eV}), \rightarrow 4 s(12.9 \mathrm{eV}), \rightarrow 4 p(13.5 \mathrm{eV}), \rightarrow 5 p(14.1 \mathrm{eV}), 1 b_{2}$ $\rightarrow 3 s(13.8 \mathrm{eV})$, and $\rightarrow 4 s(16.9 \mathrm{eV})$ excitations are reported by P. Gürtler, V. Saile, and E. E. Koch (Ref. 2), in general accord with the calculated values.

${ }^{c} \mathrm{As}$ in Table III, footnote $\mathrm{c}$, employing the threshold oscillator-strength densities of Figs. 2 (b) and

2(c). The estimated threshold contributions from the various Rydberg symmetry contributions are

$3 a_{1} \rightarrow k a_{1} ; k s-0.16, k p-0.0033, k d-0.49 ; 3 a_{1} \rightarrow k b_{2}, k p-0.063, k d-0.72 ; 3 a_{1} \rightarrow k b_{1}, k p-0.026$, $k d-0.22 ; 1 b_{2} \rightarrow k a_{1} ; k s-0.80, k p-0.16, k d-0.40 ; 1 b_{2} \rightarrow k b_{2}, k p-0.17, k d-0.93,1 b_{2} \rightarrow k a_{2}$, $k d-0.37$, all in a. u. 
and third members of the series, in particular, carry relatively large $f$ numbers, giving rise to vibrational structures in the measured photoabsorption cross section similar to that observed in the $1 b_{2}^{-1}$ PES band. ${ }^{15,18}$

The calculated $1 b_{2}-n p a_{1}$ and $n p b_{2}$ series are seen to be largely Rydberg in character $(\delta=0.7)$, although the resonance $1 b_{2} \rightarrow 3 p b_{2}$ transition is evidently lowered somewhat by a contribution from the $2 b_{2}\left(\sigma^{*}\right)$ valence orbital. Because these series are weak, they are apparently obscured in the measured cross section by other stronger series (Fig. 1), accounting for the lack of assignments in these cases.

The four calculated $1 b_{2} \rightarrow$ nd series when taken together are relatively strong, but overlap with the $n s a_{1}$ series, making spectral assignment difficult in this case. However, the calculated positions and intensities are evidently in very good agreement with the quantum-defect estimates employing the value $\delta=0$, verifying the Rydberg character of the nd series.

The three calculated partial-channel photoionization components $1 b_{2}-k a_{1}, k b_{2}$, and $k a_{2}$ are shown in Fig. $2(c)$, together with the $\left(1 b_{2}^{-1}\right)^{2} B_{2}$ sum in comparison with measured values. ${ }^{3,5}$ Of these, the $1 b_{2}-k a_{2}$ profile exhibits the familiar $2 p-k d$ behavior, whereas the $1 b_{2} \rightarrow k b_{2}$ profile apparently contains a $2 b_{2}\left(\sigma^{*}\right)$ or $\sigma \rightarrow \sigma^{*}$ contribution just above threshold, similar to the $3 a_{1}-k b_{2}$ component of Fig. 2(b). However, the $1 b_{2} \rightarrow k a_{1}$ profile differs considerably from the $2 p \rightarrow k d$ like profiles of Figs. $2(a)$ and $2(b)$ in that there is evidently a very large $1 b_{2}$ $-k s a_{1}$ threshold contribution (see also Table IV). Because of this large $1 b_{2}-k a_{1}$ threshold value, as well as that of the $1 b_{2} \rightarrow k b_{2}$ contribution, there is a noticeable discrepancy between theory and experiment in the $\left(1 b_{2}^{-1}\right)^{2} B_{2}$ cross section in the $\cong 20$ to $30 \mathrm{eV}$ interval. Comparisons of Figs. 2(a) and 2(c) suggest that the prominent feature at $\sim 18$ to $20 \mathrm{eV}$ in the measured $\left(1 b_{1}^{-1}\right)^{2} B_{1}$ channel can be attributed to possible autoionization of the $1 b_{2}-2 b_{2}\left(\sigma^{*}\right)$ contribution in the $1 b_{2}$ $\rightarrow k b_{2}$ cross section into the $1 b_{1}-k b_{1}$ continuum background. In addition, configuration mixing of the $1 b_{2}$ $\rightarrow 4 a_{1}$ intravalence excitation with $3 a_{1} \rightarrow n p$ and $n d$ series can reduce the threshold value of the corresponding $1 b_{2}$ $-k a_{1}$ photoionization cross section. This process can presumably produce neutral fragments in excited electronic states, resulting in a competition between fluorescence and autoionization, in accord with recent observations of Balmer $\beta$ emission from $\mathrm{H}_{2} \mathrm{O}$ with a threshold at $17.87 \mathrm{eV}^{3}$ Additional evidence for the production of neutral excited species in the $\sim 18$ to $20 \mathrm{eV}$ interval in $\mathrm{H}_{2} \mathrm{O}$ is provided by a prominent feature in the difference of the measured absorption and ionization cross sections. ${ }^{7}$ Finally, the situation is further complicated by the dissociative nature of the $\left(1 b_{2}^{-1}\right)^{2} B_{2}$ ionic state, which leads to production of $\mathrm{OH}^{+}$and $\mathrm{H}^{+}$fragments, as well as $\mathrm{H}_{2} \mathrm{O}^{*}$ parent ions. ${ }^{5}$

The foregoing remarks suggest that a complete quantitative understanding of photoionization in the $\left(1 b_{1}^{-1}\right)^{2} B_{1}$ and $\left(1 b_{2}^{-1}\right)^{2} B_{2}$ channels of $\mathrm{H}_{2} \mathrm{O}$ requires that the effects of configuration mixing be incorporated into the development. By contrast, the $\left(3 a_{1}^{-1}\right)^{2} A_{1}$ continuum is apparently largely one electron in nature, and is described satis- factorily by the separated-channel static-exchange approximation.

\section{4. $2 a_{1}(1 . P .=32.3 \mathrm{eV})$ spectrum}

The calculated inner-valence $2 a_{1} \rightarrow n a_{1}, n b_{2}$, and $n b_{1}$ excitations of Table $\mathrm{V}$ are seen to be generally weak,

TABLE V. $2 a_{1}$ (I. P. $=32.2 \mathrm{eV}$ ) excitation spectra in $\mathrm{H}_{2} \mathrm{O}$.

$\frac{\begin{array}{l}\begin{array}{l}\text { Present results } \\ \text { energy }(\mathrm{eV}) / f \text { number ] }\end{array} \\ 2 a_{1} \rightarrow n s a_{1} / 4 a_{1}\end{array} \text { [energy (eV)/f number] }}{\text { Defect estimates }{ }^{b}}$

$\begin{array}{ll}27.31 / 0.00935 & 26.89 / 0.00854 \\ 30.23 / 0.00149 & 30.19 / 0.00199 \\ 31.15 / 0.00089 & 31.15 / 0.00075 \\ 31.55 / 0.00025 & 31.56 / 0.00036 \\ 31.85 / 0.00021 & 31.77 / 0.00020\end{array}$

$31.85 / 0.00021$

$2 a_{1} \rightarrow n p a_{1}$

$29.62 / 0.00805$ $30.95 / 0.00030$ $31.46 / 0.00051$ $31.79 / 0.00018$

$29.63 / 0.00230$

$30.95 / 0.00078$

$31.46 / 0.00035$

$31.72 / 0.00019$

$$
2 a_{1} \rightarrow n d a_{1}
$$

$30.61 / 0.000233$

$30.76 / 0.000017$

$31.33 / 0.000155$

$31.40 / 0.000043$

$31.66 / 0.000066$

$31.71 / \cdots$

$31.98 / \cdots$

$30.69 / 0.000370$

...

$31.35 / 0.000156$

...

$31.66 / 0.000080$

...

31. $82 /$

$$
2 a_{1} \rightarrow n p b_{1}
$$

$29.47 / 0.00217$ $30.91 / 0.00084$ $31.45 / 0.00043$ $31.72 / 0.00025$

$29.63 / 0.00222$

$30.95 / 0.00075$

$31.46 / 0.00034$

$31.72 / 0.00018$

$30.78 / 0.00024$

$31.39 / 0.00014$

$31.68 / 0.00008$

$31.85 / 0.00001$

$28.77 / 0.00753$

$30.78 / 0.00095$

$31.40 / 0.00047$

$31.68 / 0.00025$

$30.51 / 0.00253$

$31.26 / 0.00102$

$31.61 / 0.00045$

$31.82 / 0.00011$

$30.69 / 0,00081$

$31.35 / 0.00035$

$31.66 / 0.00018$

$31.82 / 0.00010$

$2 a_{1}-n p b_{2} / 2 b_{2}$

29. 63/0.00205

$30.95 / 0.00070$

$31.46 / 0.00031$

$31.72 / 0.00017$

$2 a_{1} \rightarrow n d b_{2}$

$31.82 / 0.00030$

$30.69 / 0.00241$

$31.35 / 0.00102$

$31.66 / 0.00052$

${ }^{a_{A s}}$ in Table III, footnote a, employing the indicated experimentally determined vertical ionization potential (Refs. 16 and 17). Additional series similar to those given here are expected to appear converging on the higher-lying ionization potentials (Ref. 36).

${ }^{\mathrm{b}} \mathrm{As}$ in Table III, footnote $\mathrm{c}$, employing the threshold oscillator-strength densities of Fig. 3(a). The estimated Rydberg-symmetry threshold contributions are $2 a_{1} \rightarrow k a_{1}, k s-0.035, k p-0.028, k d-0.010$; $3 a_{1} \rightarrow k b_{1}, k p-0.027, k d-0.022 ; 2 a_{1} \rightarrow k b_{2}, k p-$ $0.025, k d-0.065$, all in a. u. 
with little evidence present of significant contributions to the $n s a_{1}$ and $n p b_{2}$ series from $4 a_{1}\left(\gamma^{*}\right)$ and $2 b_{2}\left(\sigma^{*}\right)$ valence orbitals, respectively. In this respect the inner-valence $2 a_{1}$ series differ from the corresponding outer-valence series described above (Tables III and IV), to which the virtual valence orbitals make noticeable contributions. Although experimental studies of excitation series in the inner-valence region of $\mathrm{H}_{2} \mathrm{O}$ are apparently unavailable at present, the calculated transition energies and $f$ numbers can be compared with quantum-defect estimates. With the exceptions of some irregularities in the $n p a_{1}$, and $n d a_{1}$ series, which may indicate mixing among the various contributing $l$ waves, the calculated staticexchange excitation series are seen to be in general agreement with the corresponding defect estimates.

In Fig. 3(a) are shown the calculated $2 a_{1} \rightarrow k a_{1}, k b_{1}$, and $k b_{2}$ contributions to the $\left(2 a_{1}^{-1}\right)^{2} A_{1}$ cross section, as well as the vertical electronic result in comparison with measured $(e, 2 e)$ values. ${ }^{5}$ The three polarization components are seen to be broad and unstructured, presumably as a consequence of the spatially compact, largely oxygen $2 s$ character of the $2 a_{1}$ orbital. Consequently,

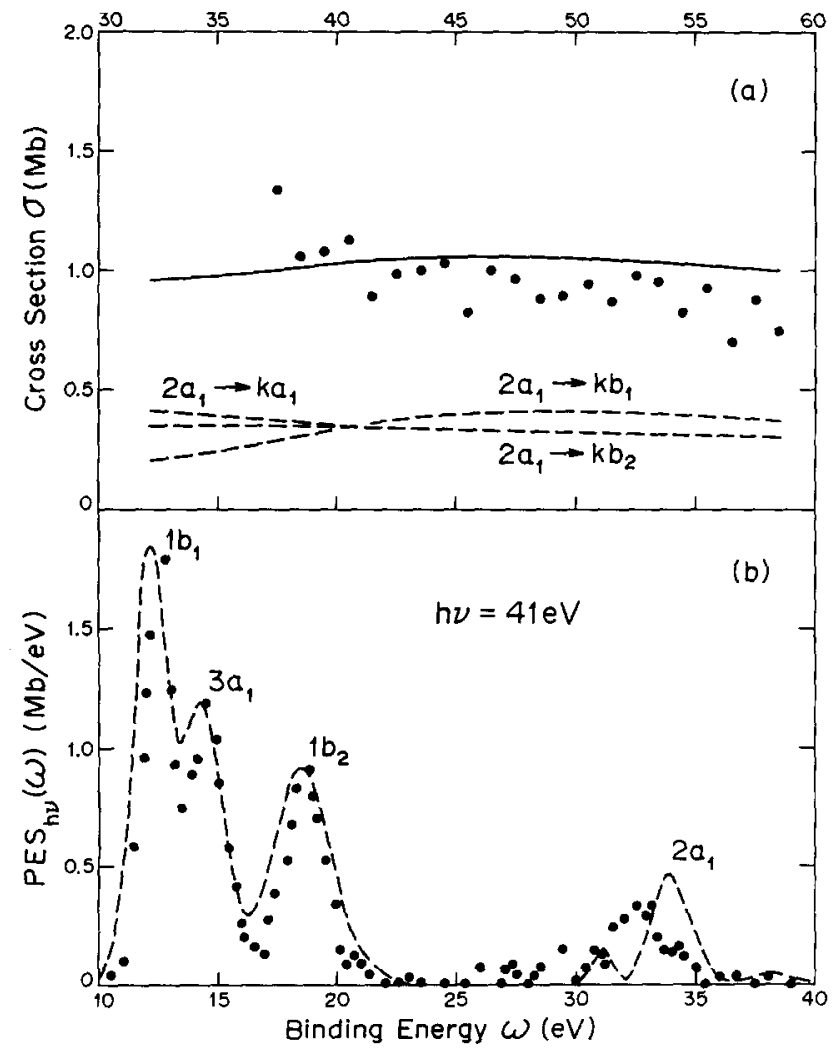

FIG. 3. (a) Inner-valence-shell $\left(2 a_{1}^{-1}\right)^{2} A_{1}$ partial-channel photoionization cross section in $\mathrm{H}_{2} \mathrm{O}$; (-) vertical-electronic staticexchange orbital cross section; (- - -) calculated polarization components as indicated; $(\bullet)$ dipole $(e, 2 e)$ measurements (Ref. 5). (b) Valence-shell photoelectron spectrum in $\mathrm{H}_{2} \mathrm{O}$ for $41 \mathrm{eV}$ incident photon energy; (-- - ) theoretical values in the intensity-borrowing approximation of Eqs. (8), obtained as discussed in the text; $(\bullet)$ dipole $(e, 2 e)$ measurements (Ref. 5), corrected for electron-analyzer transmission efficiency (Ref. 51) and normalized to the theoretical values at the peak of the $3 a_{1}^{-1}$ band. although the (out-of-plane) $2 a_{1}-k b_{1}$ component exhibits a broad, weak maximum, the $k a_{1}$ and $k b_{2}$ profiles are generally $2 s \rightarrow k p$ like in energy dependence. The calculated $\left(2 a_{1}^{-1}\right)^{2} A_{1}$ vertical electronic cross section obtained from summation of the three polarization components is evidently in generally good agreement with the experimental values. ${ }^{5}$

In spite of the good agreement obtained between theory and experiment for the $2 a_{1}$ cross section shown in Fig. $3(\mathrm{a})$, there is a modest failure of Koopmans theorem in the inner-valence region in $\mathrm{H}_{2} \mathrm{O}$, i.e., the measured $2 a_{1}$ PES band in $\mathrm{H}_{2} \mathrm{O}$ is comprised of two or more ionic states. ${ }^{5,36}$ This point is illustrated by Fig. 3(b), in which the measured valence-shell $(e, 2 e)$ photoelectron spectrum for $41 \mathrm{eV}$ incident photons is compared with theoretical values in the intensity-borrowing approximation. ${ }^{13}$ The latter spectrum is obtained from the expression $^{13}$

$$
p_{h \nu}(\omega)=\sum_{\alpha} \sigma_{\alpha}(h \nu) /\left(\omega_{\alpha} \sqrt{\pi}\right) \exp \left[-\left(\omega-\epsilon_{\alpha}\right)^{2} / \omega_{\alpha}^{2}\right],
$$

where

$$
\sigma_{\alpha}(h \nu)=\sum_{i}\left|a_{i}^{(\alpha)}\right|^{2} \sigma_{i}(h \nu)
$$

Here, $\epsilon_{\alpha}$ are the energies of the ionic states, $\omega_{\alpha}$ the corresponding widths, $\sigma_{i}(h \nu)$ the static-exchange orbital cross sections, and $\left|a_{i}^{(\alpha)}\right|^{2}$ the so-called spectroscopic factors. ${ }^{13}$ It is seen from Fig. 3(b) that the three outervalence $\left(1 b_{1}, 3 a_{1}, 1 b_{2}\right)$ PES bands in $\mathrm{H}_{2} \mathrm{O}$ are each comprised of single ionic states, in accordance with Koopmans theorem, for which the spectroscopic factors are unity. By contrast, a number of ionic states having nonnegligible spectroscopic factors contribute to the $2 a_{1}$ inner-valence band, accounting for its substantially greater width relative to the outer-valence bands. ${ }^{36}$ In each case the individual linewidths $\left.\omega_{\alpha} \cong 1 \mathrm{eV}\right)$ can be estimated from the envelopes of vibrational excitation in the Franck-Condon energy-gradient approximation, ${ }^{4-50}$ although in the present work these are simply chosen to provide a reasonable fit to the measured profile. It is seen that the measured spectrum, which includes the appropriate electron analyzer transmission-efficiency corrections, ${ }^{51}$ is in generally good agreement with the theoretical results of Eqs. (8) obtained following this procedure, although there is a modest misalignment of the peak positions.

\section{Total valence-shell cross section}

In Fig. 4 are shown the four calculated valence-shell partial-channel photoionization cross sections in $\mathrm{H}_{2} \mathrm{O}$, the corresponding total cross section, and measured total photoionization values obtained from synchrotron-radiation and $\left(e, e+\right.$ ion) electron-impact studies. ${ }^{3,5}$ The two measured photoionization cross sections of Fig. 4 include contributions from all ionic fragments produced, and, consequentiy, are appropriate for comparison with the calculated values for parent-ion production. Evidently, the measured cross sections exhibit appropriate increases at the three outer-valence thresholds, and there is even a small discernible feature present near the $2 a_{1}$ threshold in the $(e, e+$ ion $)$ data. Although the ex- 


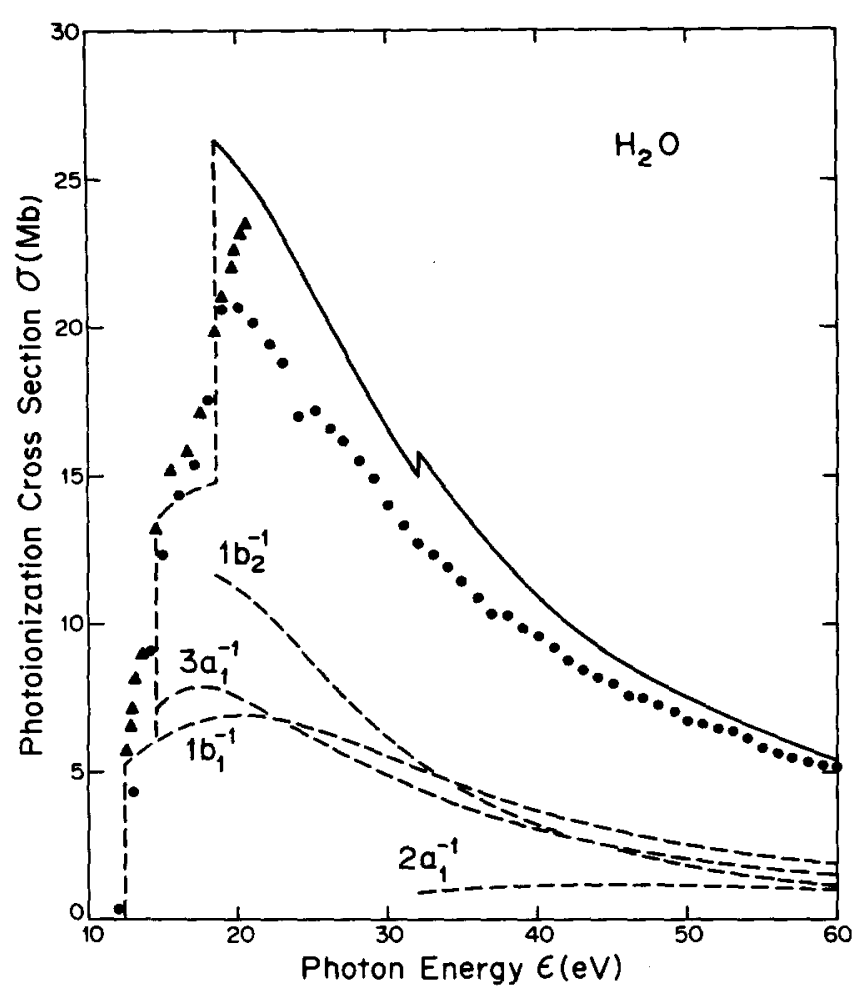

FIG. 4. Valence-shell total photoionization cross section in $\mathrm{H}_{2} \mathrm{O}$; experimental, ( $\left.\bullet\right)(e, e+$ ion) measurements (Ref. 5); ( $)$ synchrotron-radiation measurements (Ref. 3); (----) calculated partial-channel ionization cross sections as indicated; (-) total calculated vertical-electronic ionization cross section. perimental and theoretical values are in general agreement, the calculated $1 b_{2}^{-1}$ channel cross section is seen to provide an overestimate in the $\sim 20$ to $25 \mathrm{eV}$ energy interval. The effects of channel coupling and autoionization can be expected to diminish the $1 b_{2}^{-1}$ value in this energy interval, however, presumably also accounting for the structure in the synchrotron-radiation measurements of the $\left(1 b_{1}^{-1}\right)^{2} B_{1}$ profile of Fig. 2(a), as indicated above. In spite of the neglect of channel coupling in the present calculations, the total cross section obtained is seen to be in generally good agreement with the measured values, suggesting the separated-channel results provide a useful first approximation to the valence dipole spectrum in $\mathrm{H}_{2} \mathrm{O}$.

\section{Comparisons with previous calculations}

In Table VI is shown a summary of experimental outer-valence-shell assignments and excitation energies in $\mathrm{H}_{2} \mathrm{O}$ in comparison with the present calculations and with previously reported theoretical values. With the possible exception of the early estimates of Yeager et al.,${ }^{27}$ the previously reported studies, particularly those in the IVO approximation, ${ }^{20,30}$ are generally in accord with the present more comprehensive larger-basisset calculations. The latter are also seen here, as in Tables III and IV, to be in excellent accord with the experimental values. ${ }^{2}$ The largest discrepancy between measurement and the present calculations is apparently for the $1 b_{1} \rightarrow 3 s$ excitation, in which case the RPAE cal-

TABLE VI. Comparisons of theoretical and experimental excitation energies in $\mathrm{H}_{2} \mathrm{O}$.

\begin{tabular}{|c|c|c|c|c|c|c|c|}
\hline \multirow{2}{*}{\multicolumn{2}{|c|}{$\begin{array}{l}\text { Experimental assignments }{ }^{2} \\
\text { and energies }-\epsilon_{1}(\mathrm{eV})\end{array}$}} & \multirow{3}{*}{$\begin{array}{l}\text { Present results } \\
\epsilon_{i}(\mathrm{eV}) / f_{i} \\
7.24 / 0.0208\end{array}$} & \multicolumn{5}{|c|}{ Previous calculations $\epsilon_{i}(\mathrm{eV})^{\mathrm{e}}$} \\
\hline & & & \multirow{2}{*}{$\frac{\text { Refs. } 27}{7.22}$} & \multirow{2}{*}{$\frac{28}{7.30}$} & \multirow{2}{*}{$\frac{29}{7.30}$} & \multirow{2}{*}{$\frac{30}{7.61}$} & \multirow{2}{*}{$\frac{33}{7.43}$} \\
\hline $1 b_{1}-3 s$ & 7.44 & & & & & & \\
\hline $3 p a_{1}$ & 9.998 & $9.98 / 0.0065$ & 9.02 & 9.90 & 10.04 & 10.06 & \\
\hline $3 p b_{1}$ & 10.171 & $10.10 / 0.0018$ & 9.48 & 10.32 & 10.16 & 10.16 & \\
\hline $3 d a_{1}$ & 10.990 & $11.05 / 0.0064$ & 9.61 & & 11.07 & & \\
\hline $3 d a_{2}$ & 11.041 & $11.09 / 0.0052$ & & & $\cdots$ & & \\
\hline $3 d b_{1}$ & 11.057 & $11.11 / 0.0155$ & & & 11.17 & & \\
\hline $3 d a_{1}$ & 11.122 & $11.15 / 0.0028$ & & & 11.17 & & \\
\hline $1 b_{1} \rightarrow 4 s$ & 10.64 & $10.53 / 0.0001$ & & & 10.64 & & 10.80 \\
\hline $4 p a_{1}$ & 11.374 & $11.35 / 0.0025$ & & & 11.42 & & \\
\hline $4 p b_{2}$ & 11.432 & $11.37 / 0.0003$ & & & 11.48 & & \\
\hline $4 d a_{1}$ & 11.729 & $11.76 / 0.0032$ & & & & & \\
\hline $4 d b_{1}$ & 11.770 & $11.74 / 0.0053$ & & & & & \\
\hline $3 a_{1} \rightarrow 3 s$ & 9.85 & $9.88 / 0.0670$ & 9.54 & 9.80 & $\cdots$ & 9.82 & 9.55 \\
\hline $3 p a_{1}$ & & $12.24 / 0.0002$ & & & & & $\cdots$ \\
\hline $3 p b_{2}$ & & $11.53 / 0.0376$ & & & & & 11.30 \\
\hline $3 p b_{1}$ & & $12.08 / 0.0019$ & & & & & \\
\hline $3 d a_{1}$ & & $13.14 / 0.0133$ & & & & & \\
\hline $3 d b_{2}$ & & $13.13 / 0.0315$ & & & & & \\
\hline $3 d a_{1}$ & & $13.23 / 0.0094$ & & & & & \\
\hline $3 d b_{1}$ & & $13.25 / 0.0040$ & & & & & \\
\hline
\end{tabular}

${ }^{2}$ Values taken from the measurements and assignments of P. Gürtler, V. Saile, and E. E. Koch (Ref. 2). See also Ref. 6.

${ }^{b}$ Present values of Tables III and IV.

'References to previous calculations as indicated. See also Ref. 1 for citations of earlier theoretical results. The RPAE calculations of Ref. 33 have been adjusted to account for the $1.15 \mathrm{eV}$ difference between experimental and $\mathrm{HF}$ ionization potentials (see Table I). 
culations $^{33}$ are seen to be in somewhat better accord with experiment. The latter calculations also suggest relatively strong configuration mixing between the $1 b_{2}$ - $3 s a_{1} / 4 a_{1}$ excitation and other hole-particle combinations (not shown), in support of related comments made in Secs. IV A. 2 and IV A. 3. In spite of its general interest and importance, it is seen from Table VI that relatively few theoretical studies have been reported to date of the discrete dipole excitation spectrum in $\mathrm{H}_{2} \mathrm{O} .^{5 \rightarrow}$

Previously reported studies of valence-shell photoionization cross sections in $\mathrm{H}_{2} \mathrm{O}$ include those in planewave, ${ }^{31}$ one-center Coulomb, ${ }^{32} \mathrm{RPAE},{ }^{33}$ one-center inversion potential, ${ }^{34}$ and $X_{\alpha}{ }^{35}$ approximations. Although the plane-wave results are not in accord with experimental values or with the present calculations, the onecenter Coulomb and inversion-potential calculations give cross sections in generally good agreement with measured values, confirming the dominance of the oxygen atom orbitals in the photoionization of $\mathrm{H}_{2} \mathrm{O}$. Similarly, the $X_{\alpha}$ studies indicate good agreement between oxy gen atom and $\mathrm{H}_{2} \mathrm{O}$ orbital cross sections, although no comparisons with the available experimental values are reported. ${ }^{35}$ There is no evidence in any of the three onecenter calculations of contributions from $2 b_{2}\left(\sigma^{*}\right)$ or $4 a_{1}\left(\gamma^{*}\right)$ orbitals in the cross sections or in the discrete spectral regions, however. By contrast, the present calculations suggest that single-channel results should overestimate the $\left(1 b_{2}^{-1}\right)^{2} B_{2}$ cross section at threshold [Fig. 2(c)], allowing for the possibility of autoionization into the $\left(1 b_{1}^{-1}\right)^{2} B_{1}$ channel to account for the presence of a strong feature in the $\sim 18$ to $20 \mathrm{eV}$ interval [Fig. $2(a)]$. Of the previously reported theoretical studies, only the RPAE results exhibit a similar feature. Consequently, it is clear that more detailed coupled-channel calculations are in order to clarify this point, and that apparent agreement between single-channel calculations and experimental values can possibly mask important and interesting features in molecular photoionization cross sections.

\section{B. $K$-edge (I.P. $=539.7 \mathrm{eV}$ ) region in $\mathrm{H}_{2} \mathrm{O}$}

The calculated $1 a_{1} \rightarrow n a_{1}, n b_{1}$, and $n b_{2}$ excitation series are shown in Table VII in comparison with corresponding quantum-defect estimates obtained employing defects of $\delta=1.40,0.7$, and 0 for $s, p$, and $d$ series, respective. ly. As in the case of outer- and inner-valence excitations, the $1 a_{1} \rightarrow n a_{1}$ series is evidently comprised of individual interlaced $n s a_{1} / 4 a_{1}, n p a_{1}, n d\left(z^{2}\right) a_{1}$, and $n d\left(x^{2}-y^{2}\right) a_{1}$ series, and the $1 a_{1}-n b_{1}$ and $n b_{2}$ excitations include $n p b_{1}, n d b_{1}$ and $n p b_{2}, n d b_{2}$ Rydberg series, respectively. In addition, the $n b_{2}$ excitations include the now familiar $2 b_{2}\left(\sigma^{*}\right)$ contribution. Because of the wellknown sensitivity of $K$-edge $1 s$-to-valence-orbital excitations to electronic relaxation and reorganization effects neglected in the present studies, the calculated $1 a_{1}-3 s a_{1} / 4 a_{1}$ and $2 b_{2}$ transitions at 530.9 and 531.7 $\mathrm{eV}$, respectively, fall somewhat below the observed strong features at 534.0 and $535.9 \mathrm{eV}$ in the measured electron impact-excitation spectrum [Fig. 5(b)]. ${ }^{4}$ The relative intensities of the measured peaks are in general agreement with the calculated values, however. More-

TABLE VII. $1 a_{1}$ (I. P. $=539.7$ eV) excitation spectra in $\mathrm{H}_{2} \mathrm{O}$.

\begin{tabular}{ll}
$\begin{array}{l}\text { Present results } \mathrm{s}^{\mathrm{a}, \mathrm{b}} \\
\text { [energy }(\mathrm{eV}) / \mathrm{f} \text { number] }\end{array}$ & $\begin{array}{l}\text { Defect extimates } \\
\text { [energy }(\mathrm{eV}) / f \text { numberl }\end{array}$ \\
\hline
\end{tabular}

$530.91 / 0.03985$ $537.17 / 0.00114$ 538. $45 / 0.00029$ $538.95 / 0.00011$ $539.28 /$.

$$
1 a_{1} \rightarrow n s a_{1} / 4 a_{1}
$$

\section{$534.39 / 0.00269$} $537.69 / 0.00063$ $538.65 / 0.00024$ $539.06 / 0.00011$ $539.27 / 0.00006$

$$
1 a_{1} \rightarrow n p a_{1}
$$

$536.43 / 0.00861$ $538.06 / 0.00009$ $538,79 / 0.00005$ $539.23 / 0.00001$

$537.13 / 0.00019$ $538.45 / 0,00006$ $538.96 / 0.00003$ $539.22 / 0.00002$

$538.20 / 0.00011$ $538.23 / 0.00200$ $538.85 / 0.00065$ $538.87 / 0.00018$ $539.12 / 0.00007$ $539.14 / \cdots$

$$
1 a_{1} \rightarrow n d a_{1}
$$

$538.19 / 0,00167$ ...

$538.85 / 0.00070$ ...

$539.16 / 0.00036$ ...

$$
1 a_{1} \rightarrow n p b_{1}
$$

$536.13 / 0.01763$ 538. $23 / 0.00009$ $538.89 / 0.00003$ $540.30 \%$.

$537.13 / 0.00021$ $538.45 / 0.00007$ 538. $96 / 0.00003$ $539.22 / 0.00002$

$538.17 / 0.00357$ $538.85 / 0.00139$ $540.15 / 0.00060$

$538.19 / 0.00223$ $538.85 / 0.00094$ 539. $16 / 0.00048$

$531.69 / 0.07252$ $537.51 / 0.00214$ $538.59 / 0.00058$ $539.03 / 0.00024$ $539.34 / \cdots$

$$
1 a_{1}-n d b_{1}
$$$$
539.16 / 0.00048
$$

$$
1 a_{1} \rightarrow n p b_{2} / 2 b_{2}
$$

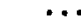

$537.13 / 0.00205$ $538.45 / 0.00070$ $538.96 / 0.00031$ $539.22 / 0.00017$

$$
1 a_{1} \rightarrow n d b_{2}
$$

$538.17 / 0.00037$ $538.87 / 0.00018$ $539.22 / 0.00002$

$538.19 / 0.00029$ $538.85 / 0.00012$ $539,16 / 0.00006$

${ }^{a}$ As in Table III, footnote a, employing the indicated experimentally determined vertical ionization potential. ${ }^{14}$

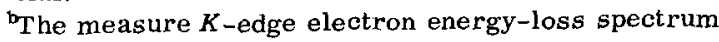
[Fig. 5(b)] is seen to include strong features at $534.0,535.9$, and $537.1 \mathrm{eV}$ that are in accord with the relative positions and strengths of the calculated $1 a_{1} \rightarrow 3 s a_{1} / 4 a_{1}, 2 b_{2}$, and $3 p b_{1}$ excitations. A fourth peak in the measured spectrum falls in the region $(538.5 \mathrm{eV})$ of many individual Rydberg transitions making specific assignment impossible.

${ }^{c}$ As in Table III, footnote $c$, employing the threshold oscillator-strength densities of Fig. 5(a). The estimated Rydberg symmetry contributions are $1 a_{1}$ $\rightarrow k a_{1}, k s-0.011, k p-0.0023, k d-0.045 ; 1 a_{1}$ $-k b_{1}, k p-0.0025, k d-0.060 ; 1 a_{1} \rightarrow k b_{2}, k p-$ $0.025, k d-0.0078$, all in a.u. 


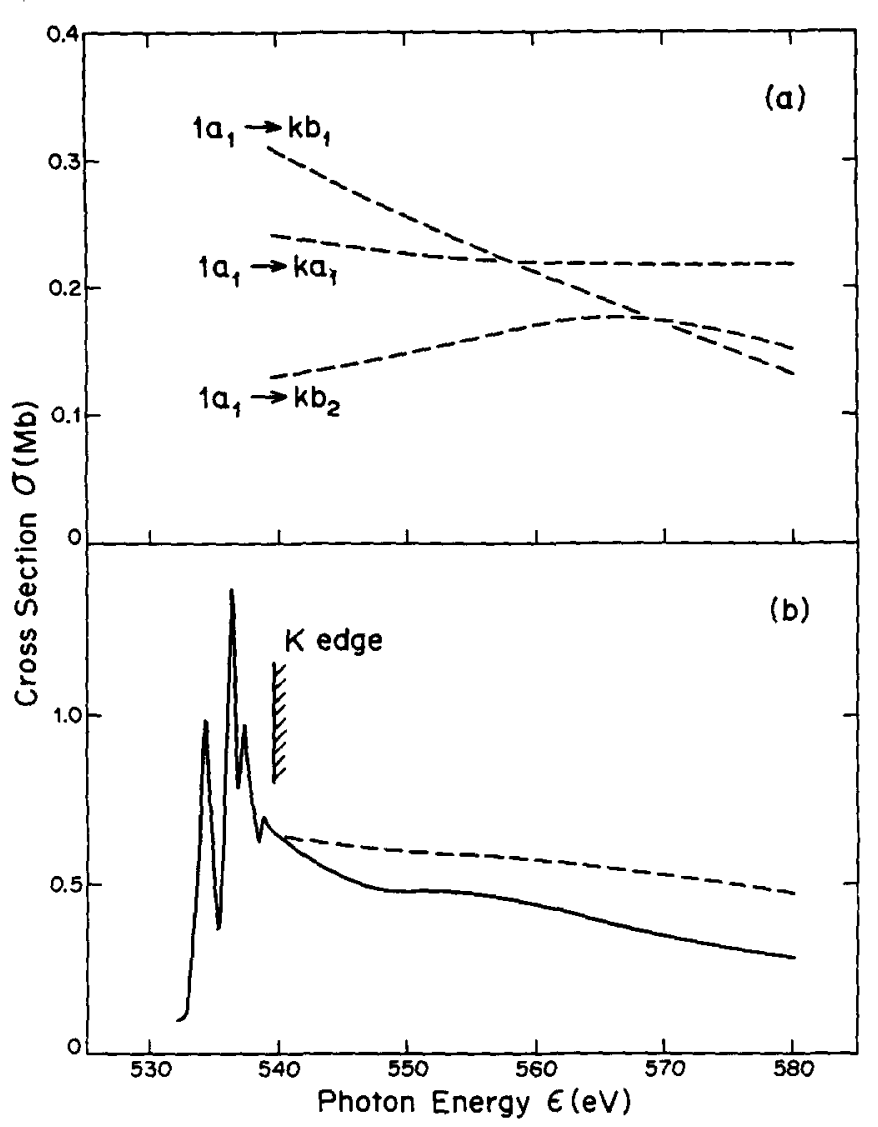

FIG. 5. (a) Calculated static-exchange $K$-edge photoionization cross sections in $\mathrm{H}_{2} \mathrm{O}$ as indicated. (b) Total $\mathrm{K}$-edge photoionization cross section in $\mathrm{H}_{2} \mathrm{O}$; (- c) calculated static-exchange results obtained from (a) above; (-) measured electron energy-loss spectrum (Ref. 4) multiplied by the factor $\left(\epsilon / \epsilon_{t}\right)^{3}$ and normalized to the calculated value at threshold.

over, the calculated $1 a_{1} \rightarrow 3 p b_{1}$ Rydberg transition, which is significantly and inexplicibly stronger than the corresponding quantum-defect estimate, is seen to be in good agreement with the third strong feature at 537.1 $\mathrm{eV}$ in the electron impact-excitation spectrum. An additional excitation peak identified in the experimental profile falls in the region of many individual Rydberg excitations ( $538.5 \mathrm{eV}$ ), so a specific assignment is not possible in this case. Evidently, the calculated excitation series of Table VII account for the strong features observed below threshold in the electron impact-excitation measurements. 5

Because the $4 a_{1}\left(\gamma^{*}\right)$ and $2 b_{2}\left(\sigma^{*}\right)$ valence orbitals contribute primarily to the discrete excitations of Table VII, presumably as a consequence of the very attractive nature of the $1 a_{1}^{-1}$ hole-state potential, the $1 a_{1}-k a_{1}, k b_{1}$, and $k b_{2}$ photoionization cross sections are expected to be largely unstructured. This expectation is verified by the calculated profiles shown in Fig. 5(a), which are seen to be generally unstructured, although there is a weak, broad maximum in the $1 a_{1}-k b_{2}$ channel. The total $K$ edge photoionization cross section obtained from the calculations is compared with measured values in Fig. 5(b). The measured energy-loss spectrum ${ }^{5}$ has been converted to cross-sectional values by multiplying with the factor $\left(\epsilon / \epsilon_{t}\right)^{3}$ and normalizing to the calculated value at threshold. Evidently, the two results are in general but not precise agreement, with the weak maximum at $\sim 555 \mathrm{eV}$ in the measured values attributable to the corresponding broad feature in the calculated $1 a_{1}-k b_{2}$ contribution.

\section{CONCLUDING REMARKS}

A great many experimental studies of photoprocesses in the water molecule have been reported to date, including recent detailed measurements of total photoabsorption and ionization cross sections, and of the partial-channel branching ratios for production of specific parent-ionic states and of dissociative ionic fragments. In view of the importance of this familiar compound in many connections, and in the absence of completely satisfactory earlier theoretical studies, a comprehensive theoretical account of the general features of the complete dipole excitation spectrum in $\mathrm{H}_{2} \mathrm{O}$ is given here.

As in previously reported studies in this series, which now includes investigations of $\mathrm{H}_{2}, \mathrm{~N}_{2}, \mathrm{O}_{2}, \mathrm{~F}_{2}$, $\mathrm{CO}, \mathrm{CO}_{2}, \mathrm{H}_{2} \mathrm{CO}, \mathrm{C}_{2} \mathrm{H}_{2}$, and $\mathrm{O}_{3}$ molecules, it is found helpful to clarify the calculated discrete excitation series and photoionization cross sections in $\mathrm{H}_{2} \mathrm{O}$ in terms of contributions from valence $4 a_{1}\left(\gamma^{*}\right)$ and $2 b_{2}\left(\sigma^{*}\right)$ virtual orbitals, and from the more diffuse orbitals that give rise to Rydberg series and corresponding hydrogen-like photoionization continua. The discrete-basis-set methodology employed is particularly well suited for this purpose, allowing for the discussion of both discrete and continuum portions of the spectrum on a common basis.

Use of very large basis sets in the present study results in highly satisfactory descriptions of intravalence and Rydberg transitions in the static-exchange or improved-virtual-orbital approximation. Specifically, the calculated $n a_{1}$ series are seen to separate into well defined $n s a_{1}, n p a_{1}$, and $n d a_{1}$ Rydberg series. The $4 a_{1}\left(\gamma^{*}\right)$ virtual valence orbital is found to contribute strongly to the $n s a_{1}$ series, particularly to the $1 b_{1}-, 3 a_{1}-$, and $1 b_{2}$ $\rightarrow 3 s a_{1} / 4 a_{1}$ resonance members, which lead to broad photodissociation bands, and which are given a mixed Rydberg/valence designation. This is in marked contrast to most earlier theoretical studies, which suggest the excited states in $\mathrm{H}_{2} \mathrm{O}$ include only Rydberg transitions. The $n b_{2}$ series are found to include $n p b_{2}$ and $n d b_{2}$ Rydberg series, as well as perturbing contributions from the $2 b_{2}\left(\sigma^{*}\right)$ virtual valence orbitals, whereas the $n b_{1}$ excitations are entirely $n p b_{1}$ and $n d b_{1}$ Rydberg-like in nature. Of particular interest in the calculations is the appearance of four $d$-type $\left[n d\left(z^{2}\right) a_{1}, n d\left(x^{2}-y^{2}\right) a_{1}\right.$, $n d b_{1}$, and $\left.n d b_{2}\right]$ Rydberg series, in excellent accord with recent detailed assignments of the $1 b_{1}$ excitations. Although the separated-channel static-exchange calculations are seen to provide a useful first approximation to the important discrete spectral features in the $\sim 7$ to $20 \mathrm{eV}$ energy interval in $\mathrm{H}_{2} \mathrm{O}$, there is evidence of configuration mixing in some cases, which require subsequent additional study. 
The calculated outer-valence-shell $\left(1 b_{1}^{-1}\right) X^{2} B_{1}$, $\left(3 a_{1}^{-1}\right)^{2} A_{1}$, and $\left(1 b_{2}^{-1}\right)^{2} B_{2}$ partial-channel photoionization cross sections are found to be in generally good agreement with corresponding synchrotron-radiation and dipole $(e, 2 e)$ measurements. In particular, the measured values for the $\left(3 a_{1}^{-1}\right)^{2} A_{1}$ channel are in very good agreement with the calculations, suggesting that the separatedchannel approximation is a good one in this case. There is evidence, however, of coupling between the $\left(1 b_{1}^{-1}\right)^{2} B_{1}$ and $\left(1 b_{2}^{-1}\right)^{2} B_{2}$ channels, in which case a strong feature in the measured $1 b_{1}^{-1}$ profile not present in the separatedchannel calculations can be attributed to autoionization of $1 b_{2} \rightarrow 2 b_{2}$ contributions in the $1 b_{2} \rightarrow k b_{2}$ continuum. Similarly, the calculated separated-channel $\left(1 b_{2}^{-1}\right)^{2} B_{2}$ cross section provides an overestimate in the $\sim 20 \mathrm{eV}$ threshold region, suggesting that this absorption strength shoulc properly appear in the $\left(1 b_{1}^{-1}\right) X^{2} B_{1}$. channel when the appropriate coupling is included in the development. When these features are accounted for, it is seen that the calculated and measured values are in good accord.

Because of a modest failure of Koopmans' theorem in the inner-valence $2 a_{1}$ region in $\mathrm{H}_{2} \mathrm{O}$, a number of final ionic states contribute to photoelectron spectra in the $\sim 30$ to $40 \mathrm{eV}$ binding-energy region, giving rise to a very broad effective $2 a_{1}^{-1}$ band. Calculations in the intensity-bor rowing sudden approximation, employing separately reported spectroscopic factors and the staticexchange $2 a_{1}$ orbital cross section, are seen to be in good agreement with measured PES and corresponding cross sections obtained from dipole $(e, 2 e)$ studies.

The total valence-shell photoabsorption and ionization cross sections obtained from the separated-channel static-exchange calculations are seen to account quantitatively for all of the important features present in the corresponding measured profiles. Specifically, the $1 b_{1} \rightarrow, 3 a_{1} \rightarrow$, and $1 b_{2} \rightarrow 3 s a_{1} / 4 a_{1}$ intravalence transitions are seen to dominate the discrete spectral interval, and increases at the various thresholds in the measured ionization cross section are attributed to corresponding features in the $1 b_{1}^{-1}, 3 a_{1}^{-1}$, and $1 b_{2}^{-1}$ ionization continua. Moreover, the weak $\left(2 a_{1}^{-1}\right)^{2} A_{1}$ contribution is seen to account for a broad structure in the measured cross section at the appropriate threshold.

Finally, the calculated $1 a_{1}^{-1} K$-edge excitation spectra are seen to clarify the origins of three strong features observed below threshold in the measured electron energy-loss spectrum. In contrast to the valence-shell spectra, the $1 a_{1}^{-1}$ hole-state potential supports lowlying $4 a_{1}\left(\gamma^{*}\right)$ and $2 b_{2}\left(\sigma^{*}\right)$ orbitals that contribute entirely to the discrete spectral region, accounting for two of the measured features, with the third peak attributed to a strong $1 a_{1}-3 p b_{1}$ Rydberg transition. The photoionization continua are correspondingly unstructured and largely monotonic, although a very weak broad feature in the measured ionization profile is clarified by the calculated cross section.

\section{ACKNOWLEDGMENTS}

It is a pleasure to acknowledge the support the of National Science Foundation, and of the Donors of the Petroleum Research Fund, administered by the American
Chemical Society. The kind hospitality provided to P.W.L. by Professor Noel Hush at the University of Sydney is also gratefully acknowledged. We thank W. von Niessen for providing calculated inner-valence-shell ionization energies and spectroscopic factors in $\mathrm{H}_{2} \mathrm{O}$, and the authors of Ref. 3 for making the results of their synchrotron-radiation studies available prior to publication.

${ }^{1} \mathrm{C}$. W. Kern and M. Karplus, "The Water Molecule," in Water-A Comprehensive Treatise, edited by F. Franks (Plenum, New York, 1972), Vol. I, Chap. 2, pp. 21-94.

${ }^{2}$ P. Gürtler, V. Salle, and E. E. Koch, Chem. Phys. Lett. 51, 386 (1977).

${ }^{3}$ O. Dutuit, I. Ito, A. Tabche-Fouhaile, P. Morin, T. Baer, P. M. Guyon, and I. Nenner, in Extended Abstracts of the Sixth International Conference on Vacuum Ultraviolet Radiation Physics (U.S. Naval Research Laboratory, Washington, D. C., 1980), Vol, II-17, private communication.

${ }^{4} \mathrm{G}$. R. Wight and C. E. Brion, J. Electron. Spectrosc. Relat. Phenom. 4, 25 (1974).

${ }^{5}$ K. H. Tan, C. E. Brion, Ph.E. Van der Leeuw, and M. J. Van der Wiel, Chem. Phys. 29, 299 (1978).

${ }^{6} \mathrm{~A}$ useful recent account of photoprocesses in the discrete spectral region in $\mathrm{H}_{2} \mathrm{O}$ is given by $\mathrm{H}$. -t. Wang, W, S. Feips, and S. P. McGlynn, J. Chem. Phys. 67, 2014 (1977).

${ }^{7}$ Earlier measurements of the photo cross sections in $\mathrm{H}_{2} \mathrm{O}$ are cited and compiled by G. D. Zeiss, W. J. Meath, J. C. F. MacDonald, and D. J. Dawson, Radiat. Res. 63, 64 (1975).

${ }^{8} \mathrm{M}$. B. Robin, Higher Excited States of Polyatomic Molecules (Academic, New York, 1974).

${ }^{9}$ P. W. Langhoff, "Stieltjes -Tchebycheff Moment-Theory Approach to Molecular Photoionization Studies, "in ElectronMolecule and Photon-Molecule Collisions, edited by T. Rescigno, V. McKoy, and B. Schneider (Plenum, New York, 1979), pp. 183-224.

${ }^{10} \mathrm{P}$. W. Langhoff, "Stieltjes-Tchebycheff Moment-Theory Approach to Photoeffect Studies in Hilbert Space," in Theory and Applications of Moment Methods in Many-Fermion Systems, edited by B. J. Dalton, S. M. Grimes, J. P. Vary and S. A. Williams (Plenum, New York, 1980), pp. 191-212.

${ }^{11}$ P. W. Langhoff, T. N. Rescigno, N. Padial, G. Csanak, and B. V. MeKoy, J. Chim. Phys.-Chim. Biol. 77, 789 (1980).

${ }^{12}$ P. W. Langhoff, N. Padial, G. Csanak, T. N. Rescigno, and B. V. McKoy, Int. J. Quantum Chem. Symp. 14, 285 (1980).

${ }^{13}$ P. W. Langhoff, S. R. Langhoff, T. N. Rescigno, J. S. Schirmer, L. S. Cederbaum, W. Domcke, and W. von Niessen, Chem. Phys. 58, 71 (1981).

${ }^{14}$ K. Siegbahn, C. Nordling, G. Johansson, J. Hedman, P. F. Heden, K. Hamrin, U. Gelius, T. Bergmark, L. O. Werme, R. Manne, and Y. Bauer, ESCA Applied to Free Molecules (North-Holland, Amsterdam, 1969).

${ }^{15} \mathrm{D}$. W. Turner, C. Baker, A. D. Baker, and C. R. Brundle, Molecular Photoionization Spectroscopy (Wiley, New York, 1970); see also A. W. Potts and W. C. Price, Proc. R. Soc. London Ser. A 236, 181 (1972).

${ }^{16}$ L. Karlsson, K. Mattson, R. Jadrny, R. G. Albridge, S. Pinchas, T. Bergmark, and K. Siegbahn, J. Chem. Phys. 62, 4745 (1975).

${ }^{17} \mathrm{~S}$. T. Hood, A. Hamnett, and C. E. Brion, J. Electron Spectrosc. Relat. Phenom. 11, 205 (1977).

${ }^{18}$ S. R. Ia Paglia, J. Mol. Spectrose. 10, 240 (1963).

${ }^{19}$ Y. Harada and J. N. Murrell, Mol. Phys. 14, 153 (1968).

${ }^{20} \mathrm{~J}$. A. Horsley and W. H. Fink, J. Chem. Phys. 50, 750 (1969).

${ }^{21}$ R. P. Hosteny, R. R. Gilman, T. H. Dunning, Jr., A. Pipano, and I. Shavitt, Chem. Phys. Lett. 7, 325 (1970). 
${ }^{22}$ T. Betts and V. McKoy, J. Chem. Phys. 54, 113 (1971).

${ }^{23} \mathrm{~J}$. N. Murrell, A. Conway, and Y. Harada, Mol. Phys. 20, 161 (1971).

${ }^{24}$ C. R. Claydon, G. A. Segal, and H. S. Taylor, J. Chem. Phys. 54, 3799 (1971).

${ }^{25}$ R. P. Hosteny, A. R. Hinds, A. C. Wahl, and M. Krauss, Chem. Phys. Lett. 23, 9 (1973).

${ }^{26} \mathrm{D}$. A. Truhlar, Int. J. Quantum Chem. 7, 807 (1973).

${ }^{27}$ D. Yeager, V. McKoy, and G. A. Segal, J. Chem. Phys. 61, 755 (1974).

${ }^{28}$ R. J. Buenker and S. D. Peyerimhoff, Chem. Phys. Lett. 29, 253 (1974).

${ }^{29}$ W. A. Goddard III and W. J. Hunt, Chem. Phys. Lett. 24, 464,755 (1974).

${ }^{30}$ N. W. Winter, W. A. Goddard III, and F. W. Bobrowicz, J. Chem. Phys. 62, 4325 (1975).

${ }^{31}$ J. W. Rabalais, T. P. Debies, J. L. Berkosky, J. -T. J. Huang, and F. O. Ellison, J. Chem. Phys. 61, 516 (1974).

${ }^{32}$ S. Iwata and S. Nagakura, Mol. Phys, 27, 425 (1975).

${ }^{33}$ G. R. J. Williams and P. W. Langhoff, Chem. Phys. Lett. 60,201 (1979).

${ }^{34}$ P. R. Hilton, S. Nordholm, and N. S. Hush, Chem. Phys. Lett. 64, 515 (1979)

${ }^{35} \mathrm{M}$. Roche, D. R. Salahub, and R. P. Messmer, J. Electron Spectrosc. Relat. Phenom. 19, 273 (1980).

${ }^{36}$ G. H. F. Diercksen, W. von Niessen, and L. S. Cederbaum, J. Chem. Phys. 67, 4124 (1977); and W. von Niessen (private communication).

${ }^{37} \mathrm{H}$. Lefebvre-Bion, C. Moser, and R. K. Nesbet, J. Mol. Spectrosc. 13, 418 (1964).

${ }^{38}$ H. P. Kelly, Phys. Rev. B 136, 896 (1964).

${ }^{39}$ P. W. Langhoff, M. Karplus, and R. P. Hurst, J. Chem. Phys. 44, 505 (1966).

${ }^{40}$ W. J. Hunt and W. A. Goddard, Chem. Phys. Lett. 3, 414 (1969).

${ }^{41} \mathrm{~L}$. C. Snyder and $\mathrm{H}$. Basch, Molecular Wave Functions and Properties (Wiley, New York, 1972).

${ }^{42} \mathrm{~T}$. H. Dunning and J. P. Hay, in Modern Theoretical Chemistry, edited by H. F. Schaefer III (Plenum, New York, 1976),
Vol. 3, Chap. 1.

${ }^{43}$ Separate calculations of photoexcitation and ionization in $\mathrm{H}_{2} \mathrm{O}$ were carried out independently at the Lawrence Livermore Laboratory (T.N.R. and C.F.B.), NASA-Ames Research Center (S. R. L.), Indiana University (P. W. L.), and the MaxPlanck-Institut für Astrophysik, Garching (G.H.F.D. and W.P.K.). The results for final-state $b_{1}, b_{2}$, and $a_{2}$ symmetry were found to be in excellent accord, whereas for $a_{1}$ finalstate symmetry only those basis sets employed at MPI, Garching, were judged to be completely satisfactory. Consequently, all results reported here refer to calculations performed at M PI, Garching, on the AMDAHL 470/V6 computer employing the program system MUNICH.

${ }^{44}$ I. N. Levine, Quantum Chemistry (Allyn and Bacon, Boston, 1974), 2nd edition.

${ }^{45}$ Spectral positions in the quantum-defect approximation are obtained from the familar Rydberg formula $\epsilon_{n}=\epsilon_{t}-13.6 \mathrm{eV} /$ $(n-\delta)^{2}$, with $\epsilon_{t}$ the ionization potential in $\mathrm{eV}$ and $\delta$ the appropriate defect. In order to construct the corresponding $f$ numbers in the Coulomb approximation $\left(f_{n}=g\left(\epsilon_{t}\right) /(n-\delta)^{3}\right.$; $g\left(\epsilon_{t}\right)$ in a.u. ], appropriate values of the photo cross sections at threshold $g\left(\epsilon_{t}\right)$ are required. Since the results of Fig. 2 refer to total threshold values, rather than to the required individual series-limit values, an approximate separation of the various contributions is required. This is accomplished in an unambiguous fashion employing the higher-lying calculated static-exchange $f$ numbers, resulting in the threshold contributions indicated in the tables.

${ }^{46}$ P. Gürtler, Diplomarbeit, Universität Hamburg, 1976.

${ }^{47}$ A. Gerwer, C. Asaro, B. V. McKoy, and P. W. Langhoff, J. Chem. Phys. 72, 713 (1980).

${ }^{48}$ W. I. Smith, J. Phys. B 1, 89 (1968); 2, I (1969).

${ }^{49}$ W. Domcke, L. S. Cederbaum, H. Koppel, and W. von Niessen, Mol. Phys. 34, 1759 (1977).

${ }^{50} \mathrm{H}$, Ågren and J. Müller, J. Electron Spectrosc. Relat. Phenom. 19, 285 (1980).

${ }^{51}$ C. E. Brion, A. Hamnett, G. R. Wight, and M. J. Van der Wiel, J. Electron Spectrose. Relat. Phenom. 12, 323 (1977). 